\title{
əCompressible EULAG Dynamical Core in COSMO: Convective-Scale Alpine Weather Forecasts
}

\author{
Michał Z. Ziemiański, ${ }^{a}$ Damian K. Wójcik, ${ }^{a}$ Bogdan Rosa, ${ }^{a}$ AND ZBigniew P. Piotrowski ${ }^{\mathrm{a}}$ \\ ${ }^{\mathrm{a}}$ Institute of Meteorology and Water Management-National Research Institute, Warsaw, Poland
}

(Manuscript received 21 September 2020, in final form 10 August 2021)

\begin{abstract}
This paper presents the semi-implicit compressible EULAG as a new dynamical core for convective-scale numerical weather prediction. The core is implemented within the infrastructure of the operational model of the Consortium for Small-Scale Modeling (COSMO), forming the NWP COSMO-EULAG model (CE). This regional highresolution implementation of the dynamical core complements its global implementation in the Finite-Volume Module of ECMWF's Integrated Forecasting System. The paper documents the first operational-like application of the dynamical core for realistic weather forecasts. After discussing the formulation of the core and its coupling with the host model, the paper considers several high-resolution prognostic experiments over complex Alpine orography. Standard verification experiments examine the sensitivity of the $\mathrm{CE}$ forecast to the choice of the advection routine and assess the forecast skills against those of the default COSMO Runge-Kutta dynamical core at 2.2-km grid size showing a general improvement. The skills are also compared using satellite observations for a weak-flow convective Alpine weather case study, showing favorable results. Additional validation of the new CE framework for partly convection-resolving forecasts using 1.1-, 0.55-, 0.22-, and 0.1-km grids, designed to challenge its numerics and test the dynamics-physics coupling, demonstrates its high robustness in simulating multiphase flows over complex mountain terrain, with slopes reaching $85^{\circ}$, and the flow's realistic representation.
\end{abstract}

KEYWORDS: Convective-scale processes; Mesoscale models; Model evaluation/performance; Nonhydrostatic models; Numerical weather prediction/forecasting

\section{Introduction}

High-resolution convection-permitting numerical weather prediction (NWP) models have become standard tools for operational regional applications (see, e.g., a review by Yano et al. 2018). The models undergo intensive development with a perspective of subkilometer horizontal grid size, also for ensemble applications. Their resolutions allowing for explicit representation of convective processes bring new scientific challenges to these efforts, as discussed by Yano et al. (2018, see also references therein).

In response to these challenges, the Science Plans of the Consortium of Small-Scale Modeling (COSMO) for 2010-14 (Arpagaus et al. 2010) and 2015-20 (Ziemiański et al. 2015) formulated a scientific strategy for all elements of the NWP production chain. The requirements for the dynamics and numerics pointed at the high accuracy of the numerical schemes, numerical stability, especially over steep orography, and computational efficiency. The strategy considered potential advantages of conservative formulations of the numerical schemes for treating strong gradients and discontinuities, pointed, for example, by LeVeque (2002, 3-5). Following that discussion, the Consortium decided to test an already existing conservative EULAG dynamical core for its possible implementation within the COSMO model.

EULAG has a relatively long history as a nonhydrostatic multiscale flow solver successfully used for research purposes,

\footnotetext{
D Denotes content that is immediately available upon publication as open access.
}

Corresponding author: Michał Z. Ziemiański, michal.ziemianski@ imgw.pl including representations of atmospheric, oceanic, stellar, and planetary processes (see, e.g., a review in Prusa et al. 2008). It was developed as a soundproof model based on the anelastic Lipps and Hemler (1982) equations. While the model also works as a semi-Lagrangian solver, its conservative flux-form Eulerian version was only considered within the COSMO project. Its results for the casting of the legacy EULAG FORTRAN 77 code (Prusa et al. 2008) into the FORTRAN 90/95 form followed by tests for idealized dry and moist flow configurations were published by Wójcik et al. (2011), Rosa et al. (2011), and Kurowski et al. (2011), respectively. The results for semirealistic and realistic flows over the Alps were published in Ziemiański et al. (2011) and Baldauf et al. (2013), respectively. The operationalized COSMO model employing the EULAG dynamical core, the COSMO-EULAG (CE), was presented in Kurowski et al. (2016).

In the meantime, a compressible EULAG dynamical core was developed (see Smolarkiewicz et al. 2014; Kurowski et al. 2014, 2015; Smolarkiewicz et al. 2016a). Its semi-implicit version allows for time steps restricted by the CFL stability condition for meteorological flows' speed (and not the sound's speed). That development led in the ECMWF to the construction of the finite-volume module of the global IFS model (IFS-FVM) (Smolarkiewicz et al. 2016b; Kühnlein et al. 2019). In COSMO, the work focused on the local-area convective-scale application of the core, and this publication documents the resulting CE prototype.

The novelty of the work lies in the successful implementation of the implicit-compressible formulation of the EULAG numerics within an operational regional convective-scale weather

Publisher's Note: This article was revised on 21 October 2021 to designate it as open access. 
prediction model. EULAG applies a unique design based on forward-in-time nonoscillatory differencing methods on A grids, significantly different from the current standards for NWP numerics, usually based on horizontally explicit vertically implicit methods on staggered C grids (Lock 2014; Mengaldo et al. 2019). The paper's novelty extends to testing the compressible EULAG numerics for fully realistic simulations of weather over complex Alpine orography, which provides a challenging environment for accuracy, computational robustness, and physics-dynamics coupling. The goal is to demonstrate not only an improvement of the forecasts resulting from the application of the EULAG dynamics but to demonstrate its suitability for potential research and future operational limited-area very high-resolution applications with horizontal grid sizes of $\mathcal{O}(100) \mathrm{m}$. The work extends the findings of Kurowski et al. (2016) that demonstrated the realistic and accurate representation of Alpine weather for an autumn case study performed with the anelastic CE at a 2.2-km horizontal grid.

The paper briefly describes the EULAG numerics, its main characteristics, and its interface with the COSMO model infrastructure. Next, the sensitivity of the CE results to the configuration of its multidimensional positive definite advection transport algorithm (MPDATA) advection procedure is analyzed using verification studies for the surface variables, cloud cover, and precipitation. It is followed by a similar comparison of the quality of the $\mathrm{CE}$ and the standard COSMO model (CRK) forecasts (the latter using the Runge-Kutta dynamical core), extended for upper-air variables. The further experiment compares the CE and CRK forecasts of the convective clouds' evolution for a weak-flow Alpine weather case study using Meteosat observations in the high-resolution visible (HRV) channel for verification.

Last, but not least, the paper discusses the CE's performance and suitability for very high-resolution convective-scale forecasts. The Alpine case study objectively compares CE forecasts of convective clouds for horizontal grid sizes of 2.2,1.1, and $0.55 \mathrm{~km}$. The study also considers the CE forecasts with horizontal grids of 0.22 and $0.1 \mathrm{~km}$ performed on smaller horizontal domains containing the highest Alpine peaks. The experiments document the CE's computational robustness for slopes' inclinations reaching up to $85^{\circ}$ and illustrate the influence of the model's resolution on the representation of the cloud field and the orographically induced vertical flows.

The paper's content is as follows: section 2 describes the semi-implicit EULAG dynamical core, and section 3 addresses basic aspects of the coupling of the core with the COSMO model infrastructure. Section 4 studies the CE sensitivity to the choice of advection routine. Section 5 compares the $\mathrm{CE}$ and CRK Alpine forecasts, while section 6 discusses the CE forecasts with increasing horizontal resolution. Section 7 concludes the article.

\section{Description of the EULAG dynamical core}

This section presents the semi-implicit compressible EULAG dynamical core following Smolarkiewicz et al. (2014), Kurowski et al. (2014), and Smolarkiewicz et al. (2016a). The model equations assume the strong conservation formulation (Clark 1977;
Wedi and Smolarkiewicz 2004, and references therein) and are cast in a curvilinear coordinate system $\mathbf{x}$. The transformation from the orthogonal coordinate system $\mathbf{x}_{c}$ (e.g., Cartesian or spherical, which will be called physical) to $\mathbf{x}$ is characterized by the Jacobian $\mathscr{G} \equiv\left(\operatorname{det}\left\{g_{j k}\right\}\right)$, where $\left\{g_{j k}\right\}$ is the covariant metric tensor in $\mathbf{x}$ and indices $j, k$ refer to the spatial dimensions, and the normalized Jacobi matrix $\mathbf{G} \equiv\left\{G_{j}^{k}\right\} \equiv\left\{\sqrt{\left(g_{c}\right)^{j j}}\left(\partial x^{k} / \partial x_{c}^{j}\right)\right\}$, where $\left(g_{c}\right)^{j j}$ are the diagonal elements of the contravariant metric tensor in $\mathbf{x}_{c}$ (no summation over $j$ is implied) (see, e.g., Prusa and Smolarkiewicz 2003).

In the curvilinear system $\mathbf{x}$ the model equations are

$$
\begin{aligned}
\frac{\partial \mathscr{G} \rho}{\partial t}+\nabla \cdot(\mathscr{G} \rho \mathbf{v})= & 0, \\
\frac{\partial \mathscr{S} \rho \mathbf{u}}{\partial t}+\nabla \cdot(\mathscr{G} \rho \mathbf{v} \otimes \mathbf{u})= & -\mathscr{G} \rho\left[\frac{\theta_{d}}{\theta_{0}} \mathbf{G} \nabla \varphi+\frac{\theta_{d}^{\prime}}{\theta_{a}} \mathbf{g}+\mathbf{f} \times \mathbf{u}\right. \\
& -\mathbf{M}(\mathbf{u})-\mathbf{F}], \\
\frac{\partial \mathscr{G} \rho \theta^{\prime}}{\partial t}+\nabla \cdot\left(\mathscr{S} \rho \mathbf{v} \theta^{\prime}\right)= & -\mathscr{G} \rho\left(\mathbf{v} \cdot \nabla \theta_{a}-H\right), \quad \text { and } \\
\frac{\partial \mathscr{G} \rho q_{i}}{\partial t}+\nabla \cdot\left(\mathscr{G} \rho \mathbf{v} q_{i}\right)= & -\mathscr{G} \rho Q_{i},
\end{aligned}
$$

where $\rho$ is the density of dry air, $\mathbf{v} \equiv \dot{\mathbf{x}}$ is the contravariant velocity in the new coordinate system $\mathbf{x}$, and $\mathbf{u}$ is the physical velocity with components defined in $\mathbf{x}_{c}$. The nabla operator $\nabla$ denotes vector of partial derivatives with respect to model curvilinear coordinates $\mathbf{x}$, and $\otimes$ is a tensor product.

Potential temperature is $\theta \equiv T\left(p_{0} / p\right)^{R_{d} / c_{p}}$, where $T$ is air temperature, $p$ is air pressure, $p_{0}$ is the reference pressure of $1000 \mathrm{hPa}, R_{d}$ is the gas constant for dry air, and $c_{p}$ is the specific heat of air at constant pressure. A generalized pressure variable $\varphi$ is based on the Exner function $\pi \equiv\left(p / p_{0}\right)^{R_{d} / c_{p}}$ in the following way. First, a hydrostatically balanced, horizontally uniform, and motionless ambient state is defined by ambient $\theta_{a}$ and $\pi_{a}$. The Exner function perturbation and the potential temperature perturbation are $\pi^{\prime} \equiv \pi-\pi_{a}$ and $\theta^{\prime} \equiv \theta-\theta_{a}$, respectively, and $\varphi \equiv$ $c_{p} \theta_{0} \pi^{\prime}$, where $\theta_{0}$ is a constant reference potential temperature.

Water species are represented via their mixing ratios $q_{i} \equiv \rho_{i} / \rho$, where $\rho_{i}$ is the density of a water species $i$. The pressure gradient and buoyancy terms of the momentum equation (2) are expressed with the density potential temperature (Emanuel 1994) $\theta_{d} \equiv \theta\left(1+q_{v} / \epsilon\right) /\left(1+\sum_{i} q_{i}\right)$, where $\epsilon \equiv R_{d} / R_{v}, R_{v}$ is the gas constant for water vapor, $q_{v}$ is the mixing ratio of water vapor, the summation goes over all water species $i$, and $\theta_{d}^{\prime} \equiv \theta_{d}-\theta_{a}$.

Finally, $\mathbf{g}$ is a vector of gravitational acceleration; $\mathbf{f}$ is the Coriolis parameter vector; $\mathbf{M}(\mathbf{u})$ represents metric forces (Christoffel terms) of the physical frame $\mathbf{x}_{c} ; \mathbf{F}$ represents the subgrid-scale forcing of momentum; $H$ represents the effects of all diabatic processes, including latent heating, diffusion, and radiation; and $Q_{i}$ represents sources and sinks of water species.

The above equations are complemented with the state equation for moist air:

$$
\varphi=c_{p} \theta_{0}\left[\frac{R_{d}}{p_{0}} \rho \theta\left(1+q_{v} / \epsilon\right)\right]^{R_{d} / c_{v}}-\phi_{a},
$$


where $\phi_{a}=c_{p} \theta_{0} \pi_{a}$, and the specific heat of air at constant volume $c_{v}=c_{p}-R_{d}$. By default, EULAG assumes deep atmosphere formulation.

\section{a. Numerical solution of the model equations}

The prognostic equations (1)-(4) can be expressed via a generalized formula

$$
\frac{\partial G \psi}{\partial t}+\nabla \cdot(\psi \mathbf{V})=G R
$$

where within the equations for momentum, entropy, and water species, $\psi$ represents components of physical velocity $\mathbf{u}$, potential temperature perturbation $\theta^{\prime}$, or water species mixing ratio, respectively. Also, $G \equiv \mathscr{G} \rho, \mathbf{V}$ is a function of advective velocity $\mathbf{V} \equiv G \mathbf{v}=\mathscr{G} \rho \mathbf{v}$, and $R$ is the rhs forcing divided by $G$. For the homogeneous continuity equation $\psi=\rho, G \equiv \mathscr{G}$ so that $\mathbf{V}=\mathscr{G} \mathbf{v}$.

The general prognostic problem (6) is numerically integrated using the second-order accurate forward-in-time template:

$$
\begin{aligned}
\psi^{n+1} & =\frac{G^{n}}{G^{n+1}}\left\{\left(\psi^{n}+\frac{\Delta t}{2} R^{n}\right)-\frac{\Delta t}{G^{n}} \nabla \cdot\left[\left(\psi^{n}+\frac{\Delta t}{2} R^{n}\right) \mathbf{V}^{n+1 / 2}\right]\right\}+\frac{\Delta t}{2} R^{n+1} \\
& \equiv \mathscr{C}\left[\left(\psi^{n}+\frac{\Delta t}{2} R^{n}\right), \mathbf{V}^{n+1 / 2}, G^{n}, G^{n+1}\right]+\frac{\Delta t}{2} R^{n+1}
\end{aligned}
$$

where indices $n$ and $n+1$ refer to consecutive instants, separated by a discrete time step $\Delta t$. The index $n+1 / 2$ refers to the instant at half of the time step. The discrete components of $\nabla$ assume standard centered second-order accurate finite-difference approximations. The term $\mathscr{A}$ is the second-order accurate flux-form MPDATA transport operator (see, e.g., Smolarkiewicz 1984; Smolarkiewicz and Margolin 1998; Smolarkiewicz and Szmelter 2005) discussed in the following subsection. Template (7) consistently links the treatment of advection and forcing by effective integration of forcing along the flow trajectory (Smolarkiewicz and Margolin 1993).

The homogeneous continuity Eq. (1) is solved for $\rho^{n+1}$ with $\mathbf{V}^{n+1 / 2}$ extrapolated from the time levels $n-1$ and $n$. The advection of $\rho$ defines the cumulative mass flux used as the advector for the remaining dependent variables. The numerical integration (7) of Eqs. (2) and (3) leads to an implicit linear problem for $\mathbf{u}^{n+1}$ and $\theta^{\prime n+1}$.

Its solution requires knowledge of $\varphi^{n+1}$. The material derivative of (5), and Eqs. (1), (3), and (4) lead to the following prognostic pressure equation, analogous to Eqs. (1)-(4):

$$
\begin{aligned}
\frac{\partial \mathscr{G} \rho \varphi}{\partial t}+\nabla \cdot(\mathscr{S} \rho \varphi \mathbf{v})= & -\mathscr{G} \rho\left[\xi \phi \frac{1}{\mathscr{G}} \nabla \cdot(\mathscr{G} \mathbf{v})+\frac{1}{\mathscr{G} \rho} \nabla \cdot\left(\mathscr{S} \rho \phi_{a} \mathbf{v}\right)\right. \\
& \left.-\frac{1}{\mathscr{G} \rho} \phi_{a} \nabla \cdot(\mathscr{G} \rho \mathbf{v})-\xi \phi \Pi\right]
\end{aligned}
$$

where $\phi=\varphi+\phi_{a}, \xi \equiv R_{d} / c_{v}$, and $\Pi=H / \theta+Q_{v} /\left(\epsilon+q_{v}\right)$, where $Q_{v}$ is a source/sink of the water vapor mixing ratio. The numerical time integration of (8) using a version of (7) leads to a second-order Helmholtz problem for $\varphi^{n+1}$. This Helmholtz equation is solved using a variational solver based on the generalized conjugate-residual method (Eisenstat et al. 1983), adapted for atmospheric modeling in the anelastic EULAG (see, e.g., Smolarkiewicz and Margolin 1994, 2000). The overall implicit problem for $\varphi^{n+1}, \mathbf{u}^{n+1}$ and $\theta^{\prime n+1}$ is solved iteratively.

With water species' presence, the pressure gradient and buoyancy forces are defined using the density potential temperature $\theta_{d}$ and its perturbation, respectively, according to (2). Evaluation of the terms at $(n+1)$ requires the mixing ratios of water species $q_{i}$ at $(n+1)$. Appropriate prognostic equations represented by (4) are numerically integrated using the following version of the prognostic template (7):

$$
q_{i}^{n+1}=\mathscr{A}\left(q_{i}^{n}+\Delta t Q_{i}^{n}, \mathbf{V}^{n+1 / 2}, G^{n}, G^{n+1}\right) .
$$

Because sources/sinks $Q_{i}$ provided by the microphysical parameterizations at time level $n+1$ are not known at the dynamic time stepping, half of the forcing $0.5 \Delta t Q^{n+1}$ required by template (7) is replaced in (9) by advection of $q_{i}^{n}$ with added full (and not half) forcing $Q_{i}$ at $n$. As a result, the effects of $Q_{i}$ are accounted for with the first-order accuracy. The forcing terms $\mathbf{F}$ and $H$ of the momentum and entropy equations provided by the model parameterizations are similarly available at $n$ and are accommodated with the first-order accuracy. The accuracy is considered sufficient for a relatively short time step of convective-scale applications of about $10 \mathrm{~s}$ or less.

\section{b. MPDATA}

MPDATA (Smolarkiewicz and Margolin 1998; Smolarkiewicz and Szmelter 2005) provides a conservative numerical solution of (7). It represents the volume integral of the divergence term in (7) via the sum of the normal fluxes of the advected variable through all faces bounding the volume of a computational cell.

MPDATA is an iterative procedure approximating the normal fluxes with the first-order accurate upwind scheme. The following step corrects the previous step's error via subsequent advection of the transported variable with specifically defined corrective velocity. Already two steps of the procedure are sufficient for its second-order accuracy, used for NWP-like applications. Further iterations are possible, and a third-order accurate scheme is available (Waruszewski et al. 2018).

MPDATA was originally positive definite but, currently, can be used for the physical fields of variable signs. So-called basic MPDATA applies a modified definition of the corrective velocity ensuring its general boundedness (Smolarkiewicz and Margolin 1998). Alternatively, a constant $c$ is added to the advected variable for its global positive definiteness, and the asymptotic version of such scheme with $c \rightarrow \infty$ is called gauge MPDATA (Smolarkiewicz and Clark 1986; Smolarkiewicz 2006). The basic scheme is sign preserving, and the gauge is generally not. 
MPDATA does not, in general, preserve monotonicity and its nonoscillatory options were developed by Smolarkiewicz and Grabowski (1990); see also Smolarkiewicz and Szmelter (2005). They limit the corrective fluxes in the spirit of the flux-corrected transport algorithm (Zalesak 1979). The nonoscillatory schemes are sign preserving (Smolarkiewicz and Margolin 1998): the basic one exactly and the gauge one to the machine precision. For the NWP applications, the nonoscillatory versions are recommended and are used for the current study. For general purposes, a diverse library of MPDATA versions is available (Jaruga et al. 2015).

The implicit diffusion of MPDATA is flow-gradient dependent (Piotrowski et al. 2009) and differs for the basic and gauge schemes (Smolarkiewicz and Clark 1986; Domaradzki et al. 2003; Strugarek et al. 2016).

\section{c. EULAG robustness}

Many atmospheric models applying terrain-following coordinates (Gal-Chen and Somerville 1975) experience problems while treating steep orographic slopes. Zängl (2012) considers the issue analyzing the role of the discretizations of pressure gradient force. A well-known advantage of EULAG is its stability for such problems. It was demonstrated, for example, by Smolarkiewicz et al. (2007b) for a large-eddy simulation (LES) of a flow past a complex building, by Kurowski et al. (2016) for atmosphere-at-rest experiments with the Gaussian and Schär mountains (Schär et al. 2002), or by Wedi and Smolarkiewicz (2004) for idealized flow over the Schär mountain.

The numerical design of the EULAG dynamical core focuses on the overall consistency of the scheme so that its desirable numerical properties, including computational robustness, subsume many aspects of the design. The consistency involves appropriate analytic formulation of the model. So, the perturbation form of the model equations prevents polluting the ambient state balances with truncation errors of the simulated scales (Prusa et al. 2008; Smolarkiewicz et al. 2014). The coordinate transformation is reversible and extends the standard geometric conservation law to the multicomponent tensor identity (Smolarkiewicz and Prusa 2005; Prusa et al. 2008). The implicit integration of the rhs forcings leads to the elliptic Helmholtz equation for $\varphi^{n+1}$, consistent with the internal energy constraint (Smolarkiewicz et al. 2019). That enables large time steps, unconstrained by the propagation of acoustic, gravity, and rotational modes, by suppressing the truncation errors of the explicit acoustic schemes (Smolarkiewicz et al. 2016a).

There is also the consistency between the numerical and analytical formulation of the model. The collocated data arrangement enables analytic derivation of the Helmholtz pressure equation consistent with the model discretization in the exact projection spirit. That eliminates an adverse influence of the truncation errors of the pressure problem on the entire model solution (Smolarkiewicz et al. 1997). Moreover, boundary conditions imposed on normal velocity components along the curvilinear surfaces are semi-implicit and free of shallow-slope approximations, and therefore exact for impermeable boundaries (Smolarkiewicz et al. 2007b).

The consistency within the model numerics involves the formulation of MPDATA with its corrective velocity compensating the dissipative errors of both forward-in-time and upwind schemes. MPDATA assures boundedness of the energy norm in the transport of the dependent variables (Smolarkiewicz and Prusa 2002). This ensures the model's nonlinear stability with respect to advection and aids the physical realizability of the solutions by preventing spurious intersections of simulated flow trajectories (Smolarkiewicz and Pudykiewicz 1992). Also, the implicit LES properties of the advection scheme contribute to the stability of the dynamical core (section 4).

\section{EULAG dynamical core within COSMO model infrastructure}

The semi-implicit dynamical core of EULAG is implemented within the infrastructure of the mesoscale NWP model of COSMO (Baldauf et al. 2011), version 5.05. It replaces the standard split-explicit Runge-Kutta (RK) dynamical core (Klemp and Wilhelmson 1978; Wicker and Skamarock 2002; Doms and Baldauf 2018). The CE uses the standard COSMO parameterizations of physical processes (Doms et al. 2018). They include a one-moment microphysics scheme (Reinhardt and Seifert 2006) with rain, ice, snow, and graupel, RitterGeleyn radiation scheme (Ritter and Geleyn 1992), 2.5-moment turbulence scheme with turbulent kinetic energy (TKE) advection (Mellor and Yamada 1982; Raschendorfer 2001), and multilevel soil model TERRA (Heise et al. 2003). The CE applies the native COSMO coordinate system consisting of rotated latitude-longitude coordinates in horizontal and hybrid terrainfollowing coordinates in vertical (Doms and Baldauf 2018), one of many possible grid configurations.

The differences between compressible EULAG and the RK dynamical cores involve both analytical formulations of the underlying equations and the methods of their numerical integration. For the coupling, the important differences concern the definitions of the model variables and their computational grids. EULAG variables are $\rho, \mathbf{u}, \theta, p$, and $q_{i}$, all collocated at the mass points of the A grid. RK core uses $\mathbf{u}$, $T, p$, and $m_{i}$, where $T$ is temperature and $m_{i}$ are specific measures of water species $i$, on the $\mathrm{C}$ grid with staggered velocity components.

Following Kurowski et al. (2016), the coupling uses an interface for conversion between the EULAG and RK variables and grids. To facilitate the coupling, positions of the CE's mass points follow the positions of the RK's mass points. Only velocity components are interpolated linearly between the EULAG and RK grids and vice versa (see Fig. 1 for comparison of RK and EULAG grids). At the mass points, the RK thermodynamic variables and specific measures of water species are recalculated to the EULAG thermodynamic variables and mixing ratios, and vice versa.

The COSMO $\mathrm{C}$ grid has the lowest mass level elevated over the ground at about $10 \mathrm{~m}$ altitude, and the lowest staggered level for the vertical velocity is on the ground. On that level, the homogeneous (or kinematic) boundary condition for the vertical velocity is defined for flat (or inclined) terrain, respectively. The anelastic CE (Kurowski et al. 2016) had an additional mass level on the ground to support that formulation. The compressible EULAG dynamical core lacks that 


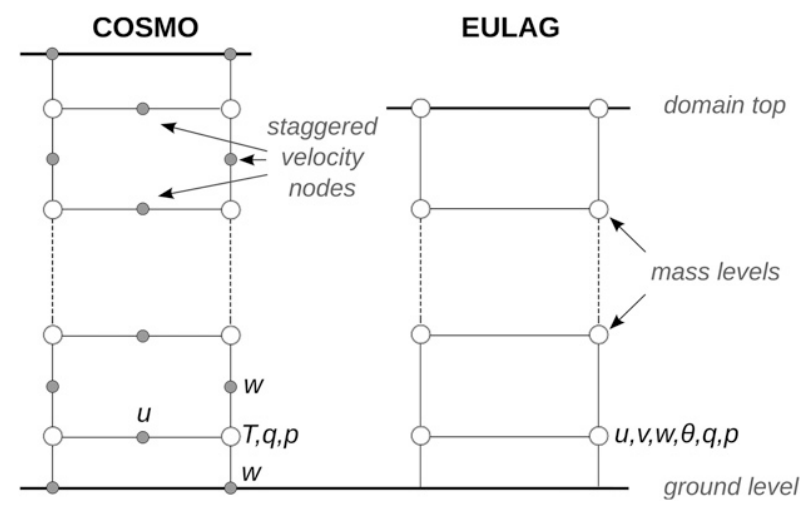

FIG. 1. Vertical structure of the computational grid in the (left) Runge-Kutta and (right) EULAG dynamical cores.

ground level to alleviate problems with defining other flow variables there. The homogeneous or kinematic boundary conditions for the vertical velocity are implemented on the lowest CE level equivalent to the COSMO lowest mass level. The tests showed that this setup does not adversely influence the performance of the model.

The novelty of CE physics-dynamics coupling, in comparison with the CRK setup, follows from the EULAG's basic idea of integration of forcings along the flow trajectory according to (7) and (9). That also concerns the forcings induced by physical parameterizations. Practical implementation of that functionality in $\mathrm{CE}$ did not require intervention within the parameterizations' codes but a consistent definition and collection of the forcings they induce. The already existing COSMO functionality to collect the temperature forcings was used and extended for water species with the help of the COSMO tracer module (Roches and Fuhrer 2012). For consistency, the TKE advection of the turbulence scheme is performed with the MPDATA, like all other model variables.

The main scientific challenges concerned the consistent treatment of the thermodynamic variables (including density and pressure as new prognostic variables) in the presence of the fully complex realistic initial and boundary conditions of the limited-area domain and its influence on the performance of the Helmholtz problem solution.

Currently, the computational time necessary for a single time stepping of the CE is $12 \%$ longer than for the CRK. With the CE's Eulerian advection requiring shorter time steps (12 s for the CE vs $20 \mathrm{~s}$ for CRK at 2.2-km horizontal grid size), the CE's overall computational time is $87 \%$ longer than for the CRK. The experiences with IFS-FVM (Kühnlein et al. 2019) indicate that a significant improvement is possible with implementing measures like split-MPDATA and adaptive time stepping.

\section{Model sensitivity to choice of advection routine}

Nonoscillatory versions of basic or gauge MPDATA can be used operationally, and a verification experiment was performed to assess the sensitivity of the forecasts to that choice. The configuration of the $\mathrm{CE}$ follows the operational configuration of the CRK, including its standard physical parameterizations. The $\mathrm{CE}$ has a horizontal grid size of $2.2 \mathrm{~km}$ and 60 vertical levels of stretched hybrid terrain-following coordinate, reaching an altitude of $22.5 \mathrm{~km}$. The radiation scheme is activated every $6 \mathrm{~min}$, and a parameterization of shallow convection is turned off.

Forecasts of $48 \mathrm{~h}$ starting daily at 0000 UTC are recalculated for June and November 2013 with the CE using basic or gauge MPDATA to provide monthly verification statistics for different thermal and circulation conditions. The horizontal Alpine domain of the operational regional NWP model of MeteoSwiss (Fig. 2) is used. The contemporary operational initial and boundary conditions are provided by MeteoSwiss.

The verification statistics are calculated using Verification System Unified Survey (VERSUS): the dedicated verification software of COSMO (Raspanti 2009). Further, the statistical significance of the score differences was evaluated for $95 \%$ confidence level following Jensen (2017) based on Nicholls (2014, 2016). The significance depends on the errors of the scores. However, for highly correlated results of the alternative methods, the scores' differences are statistically significant even if they are smaller than the sum of the scores' errors, which is the case here.

Figure 3 compares the mean errors (ME) and the rootmean-square errors (RMSE) of the forecasts of model variables verified against surface observations (SYNOP). ${ }^{1}$ While the advection choice has a very small impact on RMSE (except an improvement of 2-m temperature in June for the basic scheme), the basic MPDATA systematically improves the bias in June and November for 10-m wind speed, 2-m temperature, and total cloud cover, and in November for 2-m dewpoint temperature. The choice of advection has an impact close to neutral for mean sea level pressure (MSLP) bias in June. In November, basic MPDATA tends to improve it during the second day of the forecast but worsens during its first day. For a 2-m dewpoint temperature in June, the basic scheme tends to diminish the negative bias of the gauge scheme and to increase this bias when it is positive. The analyzed positive impact of the basic MPDATA is statistically significant.

The precipitation forecasts are compared for 3-month summer (June, July, August) and autumn (October, November, December) periods of 2013 for high statistical significance of verification results, also for strong precipitation. MeteoSwiss 1-hourly regional analyses were used as the forecasts' boundary conditions, which is sufficient for the comparison. The surface precipitation is accumulated within four 12-hourly forecasting periods and is categorized into three classes with lower bounds at $0.1,1$, and $10 \mathrm{~mm}$. Verification statistics are shown in Fig. 4 using the performance diagrams (Roebber 2009) presenting probability of detection (PoD) and success ratio [SR $=1-$ false alarm ratio (FAR)]. It allows interpretation of the results in terms of

\footnotetext{
${ }^{1}$ The verification uses the nearest model grid, except the precipitation and cloud cover, for which the model data are averaged within the radius of 15 and $30 \mathrm{~km}$ from the stations' locations, respectively.
} 

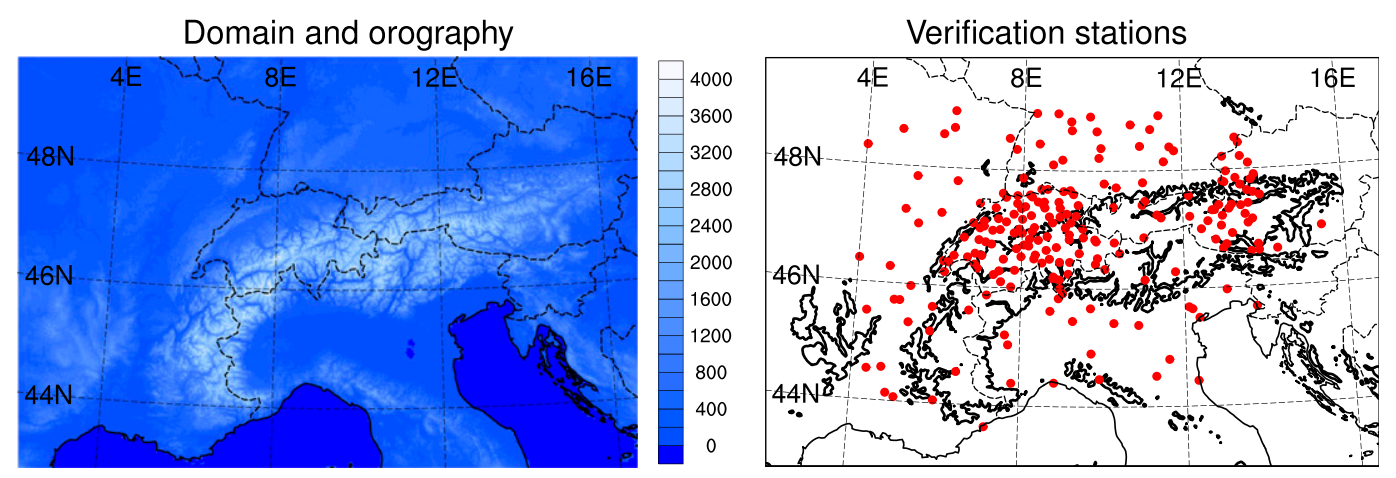

FIG. 2. (left) Extent and orography (m MSL) of the entire Alpine domain and (right) positions of observation stations used for standard verification experiments with the contour of 1-km altitude MSL.

frequency bias and critical success index (CSI) (Schaefer 1990), the latter being a ratio of a number of correct forecasts of an event to a sum of its occurrences and false forecasts of its occurrence. For the perfect forecast, the PoD, SR, CSI, and frequency bias are 1 . The diagram also shows the statistical significance of the scores' differences. The errors of the scores, necessary for evaluation of the significance, were calculated using the R library (R Core Team 2015) bootstrapping.

Figure 4 indicates that for the classes of weak (above $0.1 \mathrm{~mm}$ ) and moderate (above $1 \mathrm{~mm}$ ) precipitation, the basic MPDATA systematically diminishes the excessive frequency bias of the gauge MPDATA in summer and, to a smaller degree, in autumn. The differences are statistically significant and tend to be smaller for the moderate precipitation. The differences in the CSI are much smaller and usually nonsignificant statistically. For the strong (above $10 \mathrm{~mm}$ ) precipitation, the differences are statistically nonsignificant in summer and for most of the forecasting periods in autumn, except the 36-h forecast where the basic MPDATA diminishes the PoD slightly.

Overall, the basic MPDATA gives systematically better verification statistics for most near-surface variables, total cloud cover, and small to moderate precipitation. That strongly supports its use as the default option for operational convectivescale applications. Consequently, only the basic MPDATA is used for further experiments in the spirit of previous EULAG simulations of complex turbulent flows (e.g., Strugarek et al. 2016; Warn-Varnas et al. 2007).

The cone advection test by Smolarkiewicz and Clark (1986) helps to consider likely reasons for that superiority of basic MPDATA for the NWP-type application. It shows smaller implicit diffusivity of the gauge scheme relative to the basic one, resulting from partial compensation of the scheme's thirdorder spatial errors.

The smoother solutions of the basic scheme may diminish the verification errors via the so-called double penalty effect (Rossa et al. 2008). It promotes spatially smooth solutions over sharper and containing small-scale details with their inevitable phase errors. If so, the effect would mainly influence the perturbations of model variables rather than their mean values and reduce mainly the RMSE rather than the ME. On the contrary, Fig. 3 shows systematically decreased biases for most of the model variables with prevailing near-neutral impact on their RMSE.

That suggests a positive impact of the larger implicit diffusivity of basic MPDATA on the model's representation of the subgrid-scale (SGS) physics. That generic MPDATA's property was already documented by Margolin et al. (1999). They demonstrated that the diffusive properties of MPDATA well represent the physical SGS effects for LES simulations of a convective planetary boundary layer. Smolarkiewicz and Prusa (2002) point further at the scheme's self-adaptivity and flowgradient dependence with stronger implicit diffusion for rough and weaker for smooth resolved flows. That property contributes to the numerical robustness of EULAG, and its physical aspects are used for implicit LES (ILES) methods (Grinstein et al. 2007; Smolarkiewicz et al. 2007a).

We hypothesize that the potential larger implicit diffusivity of basic MPDATA allows for more efficient use of its ILES properties in augmenting the CE's representation of the SGS physics and gives better verification scores. The comparison of energy spectra for CE using both schemes does not contradict that hypothesis. Figure 5 shows the spectra averaged over 500, 400, and $300 \mathrm{hPa}$ for forecasts starting between 10 and 15 June and between 10 and 15 November. The spectra have a typical pattern (see Skamarock 2004) but the basic scheme's larger diffusivity, increasing with the diminishing horizontal scale, causes some deviation from the $k^{-5 / 3}$ inclination of its spectra. The gauge scheme is less diffusive, and there is no such deviation in the main part of its spectrum in November and a slight one in June.

\section{Comparison of CE and CRK}

\section{a. Standard verification tests}

This section compares the performance of the CE employing the basic MPDATA with the benchmark operational CRK model. The experiment is analogous to the one described above, uses the same verification types and periods, model domain, and boundary conditions, and additionally considers monthly verification of upper-air variables. The CE and CRK have the same configuration and resolution and use the same physical parameterizations.

The CE and CRK forecasts for June and November verified against SYNOP observations are compared in Fig. 6. The further 

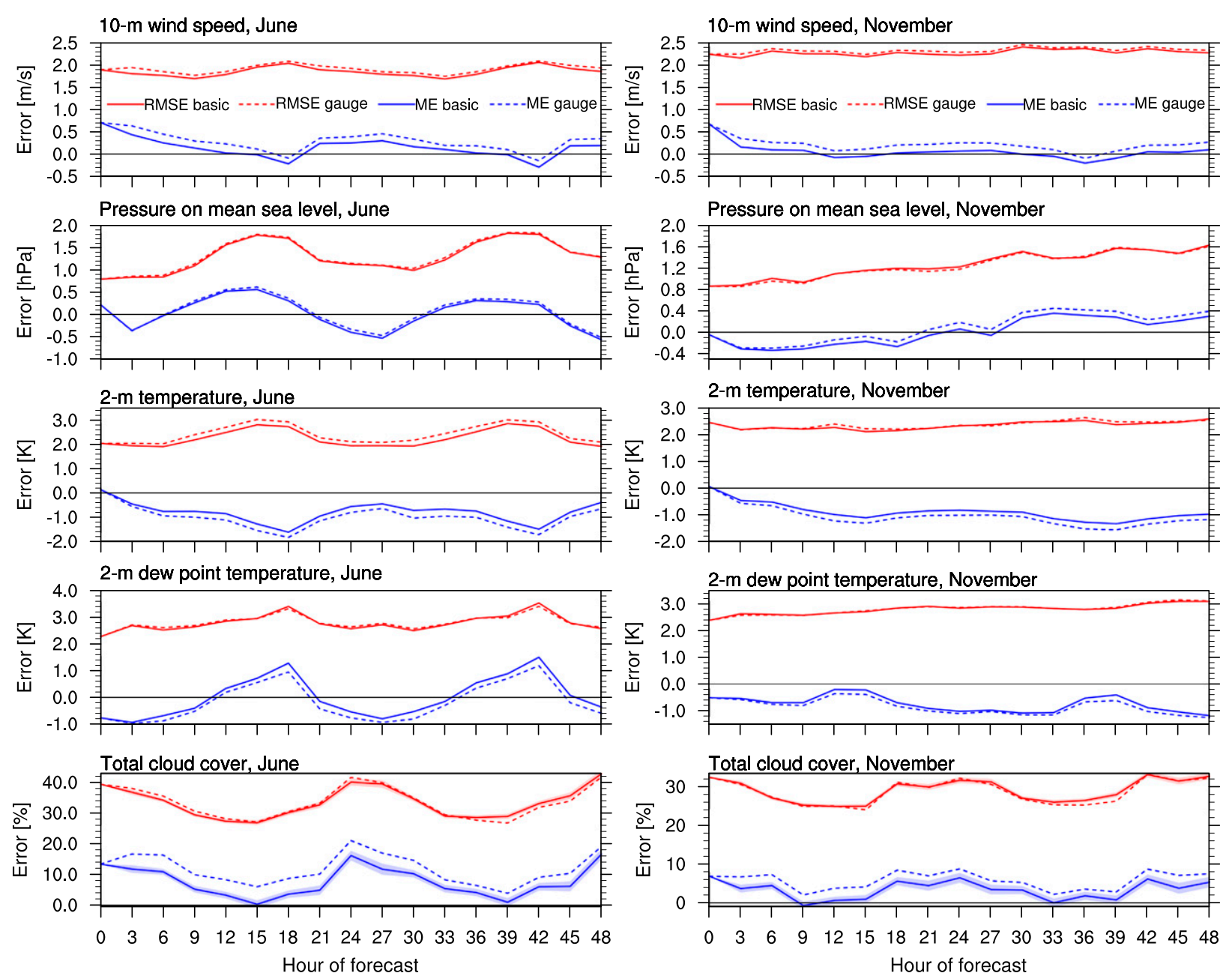

FIG. 3. RMSE (red) and ME (blue) for 48-h CE forecast with basic MPDATA (continuous line) and gauge MPDATA (dashed line) in (left) June and (right) November for near-surface variables: (top row) 10-m wind speed, (second row) pressure reduced to mean sea level, (third row) 2-m temperature, (fourth row) 2-m dewpoint temperature, and (bottom row) total cloud cover. The statistical significance of the error differences is indicated as a shaded belt encompassing the characteristics of the basic MPDATA: if the line characterizing the gauge MPDATA lies outside the belt, the differences are statistically significant with $95 \%$ confidence. For most of the variables, the belt's width is smaller than the width of the graph line.

analysis focuses on the impact of the EULAG dynamical core on the scores. In June, the impact on the RMSE is close to neutral or slightly positive, with a stronger improvement for 2-m temperature. The impact on the ME is overall positive, especially for the total cloud cover, 2-m temperature, and wind speed, and close to neutral for dewpoint temperature. The ME of CE MSLP exhibits a diurnal cycle absent in the CRK forecast. In November, the impact on the RMSE is prevailingly close to neutral, with a slightly positive impact for pressure. The impact on the ME is overall positive for MSLP, 2-m temperature, and cloud cover and neutral for wind and dewpoint temperature. The improvement of the CE scores is statistically significant.

CE and CRK upper-level forecasts of wind speed and temperature at the main pressure levels for June and November 2013 are verified against TEMP observations. Their ME, RMSE, and their statistical significance are shown in Fig. 7. Overall, the impact of the CE implementation on the scores is prevailingly neutral to positive. The main exceptions concern the temperature in the troposphere-stratosphere mixing zone and some aspects of a boundary layer representation: in June, the ME of wind speed and temperature deteriorates slightly at $850 \mathrm{hPa}$ during the day. However, a further improvement is likely with improved interrelation between the model's implicit and explicit diffusions.

Figure 8 shows the performance diagrams comparing the $\mathrm{CE}$ and CRK precipitation scores for the accumulation periods and amounts from the previous experiment. Similar to the comparison of the MPDATA versions, the CE employing basic MPDATA systematically and statistically significantly reduces an excessive frequency bias of the CRK forecast of weak and moderate precipitation for summer and autumn periods. The effect tends to be slightly smaller for autumn. The CSI is practically unchanged or slightly 


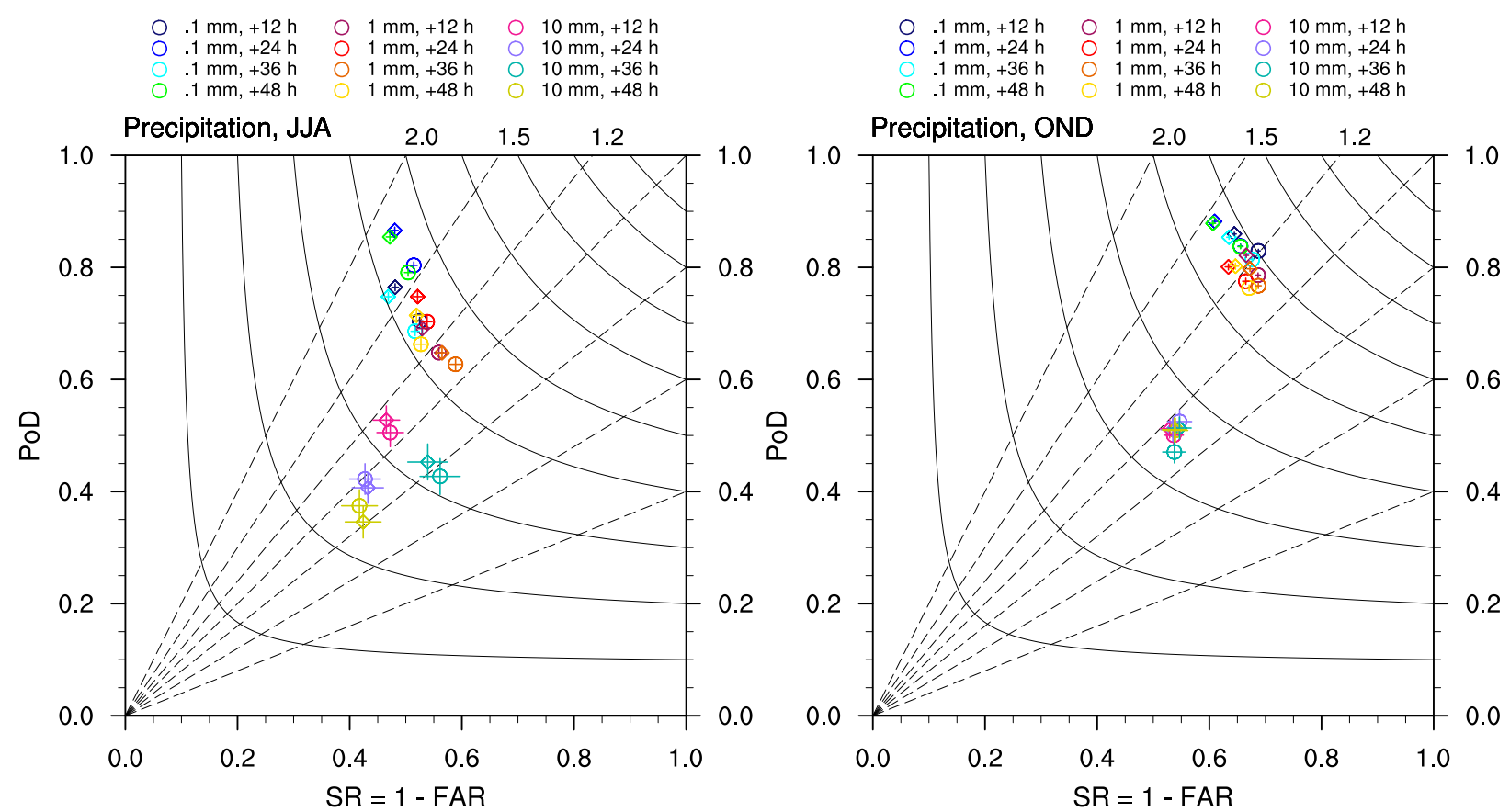

FIG. 4. Performance diagrams for 48-h CE forecast of surface precipitation with basic (circles) and gauge MPDATA (diamonds) for (left) June-August and (right) October-December. The precipitation is accumulated within 12-h periods and categorized into classes with lower bounds at $0.1,1$, and $10 \mathrm{~mm}$. The bottom axes show the SR and the left axes the PoD; solid curves of constant CSI are labeled on the right margin while straight dashed lines of constant frequency bias are labeled on the right and top margins of the figure. The statistical significance of the differences between the verification statistics is shown using crosses: if the analogous statistics lie outside the range of their crosses, the differences are statistically significant with $95 \%$ confidence.

improved. For strong precipitation, there is a statistically significant improvement of the frequency bias for 24- and 48-h forecasts in summer but also a statistically significant decrease in PoD for 36-h forecast in autumn (the latter effect is similar to the difference between the CE forecasts employing the basic and gauge MPDATA). Otherwise, the differences are statistically insignificant.

In summary, the implementation of the EULAG dynamical core improves or has a neutral effect on the verification statistics for most of the analyzed model variables. The improvement is
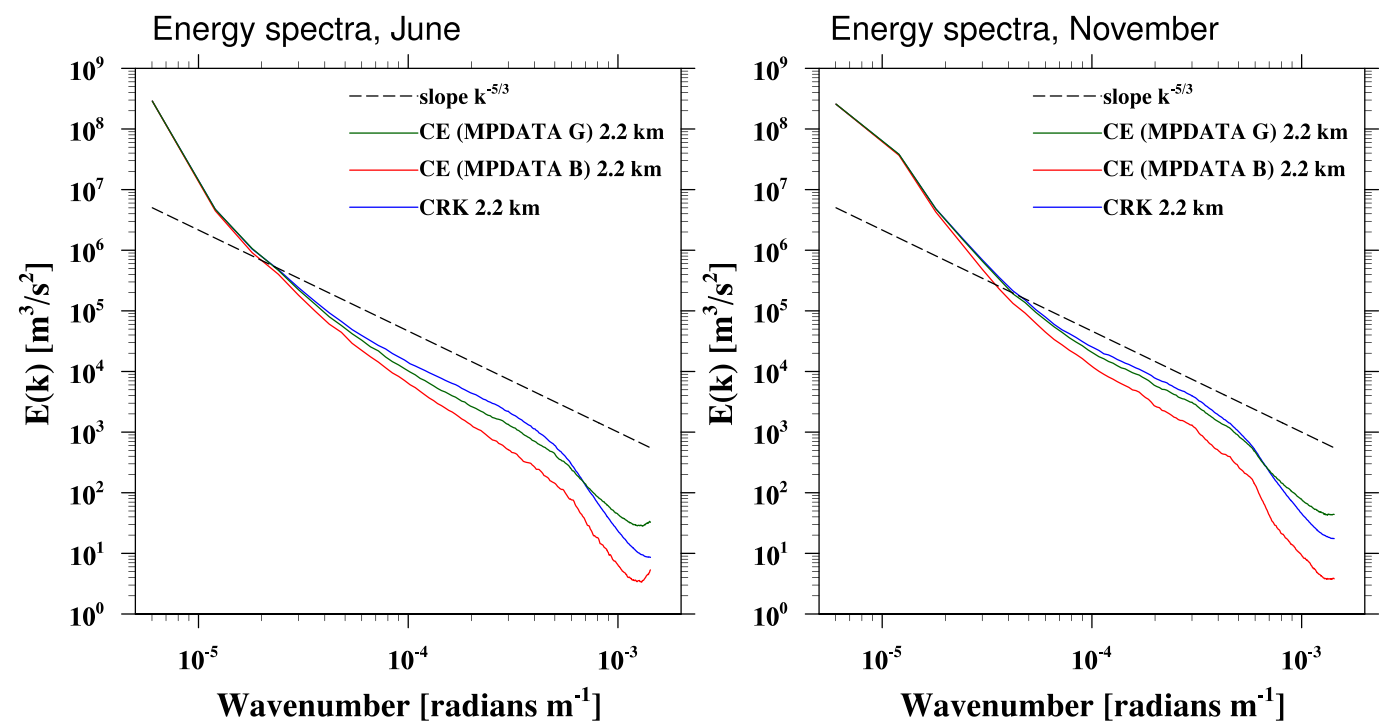

FIG. 5. Energy spectra for CE employing basic or gauge MPDATA and for CRK, for (left) June and (right) November; MPDATA B stands for basic MPDATA, and MPDATA G stands for gauge MPDATA. 

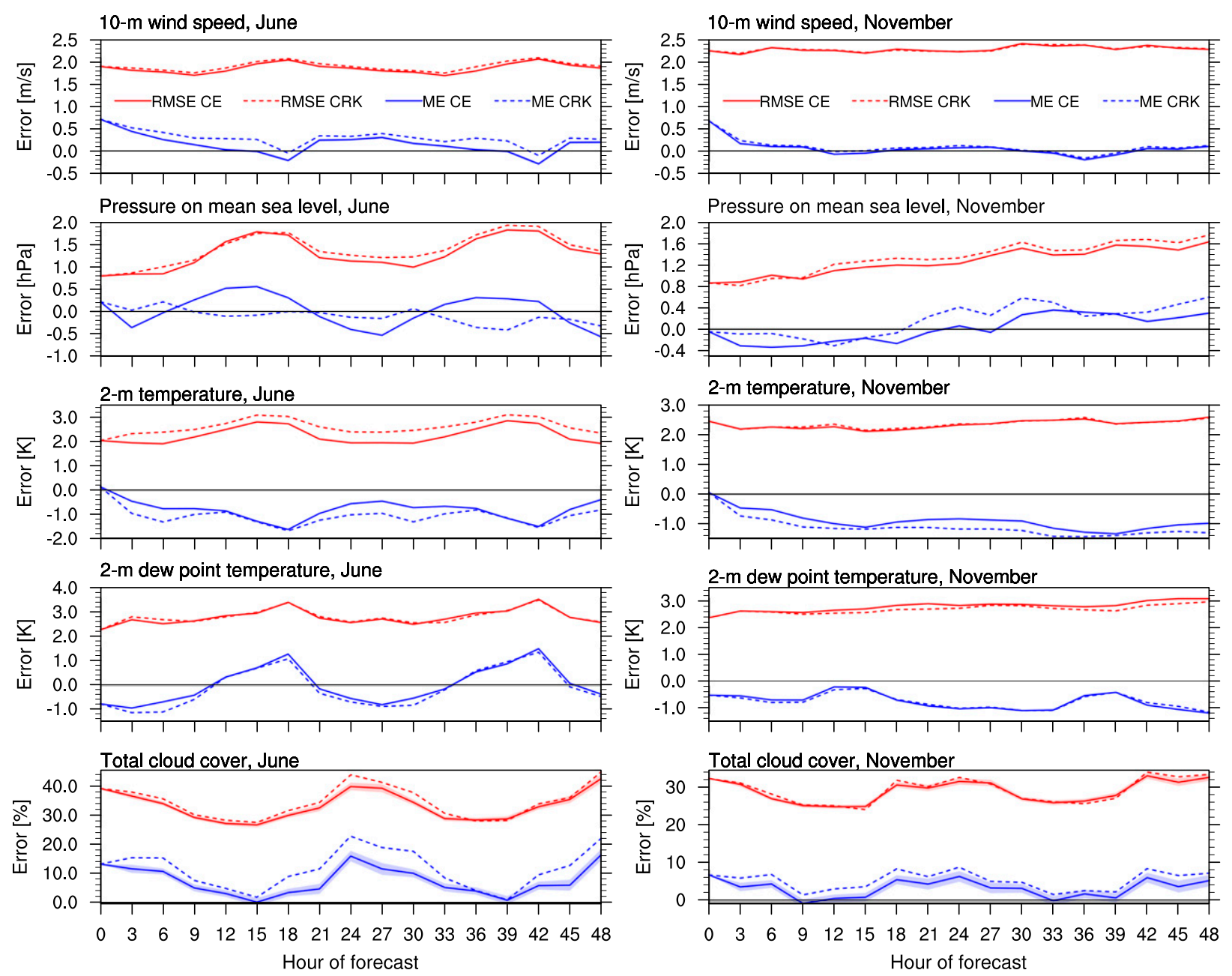

FIG. 6. As in Fig. 3, but for comparison of CE (continuous line) with CRK (dashed line).

especially prominent in June, characterized by convectively dominated weather, and it also concerns clouds and precipitation. As the forecast's quality strongly depends on the physical processes' parameterizations (see, e.g., Langhans et al. 2019), and the COSMO parameterizations were not retuned for the $\mathrm{CE}$, the results are encouraging and indicate the space for further improvement.

The differences in verification statistics between CE and CRK are similar to the differences between the basic and gauge MPDATA, while the comparison between $\mathrm{CE}$ using gauge MPDATA and CRK shows overall a smaller improvement (not shown). That suggests that the stronger ILES properties of basic MPDATA also significantly contribute to the CE's improvement over CRK. Also, the CE's smaller improvement in autumn, compared with summer, can be - at least partly_related to the ILES properties of MPDATA because they tend to be weaker for the shear-driven boundary layers (Moin and Kravchenko 1998; Brown et al. 2000; Smolarkiewicz and Prusa 2002).

Figure 5 shows that $\mathrm{CE}$ with the basic MPDATA is more diffusive than CRK, but the difference between diffusivities of CRK and CE applying the gauge scheme is smaller, especially in November. At the smallest scales, the CE with the gauge scheme is even less diffusive than the CRK. For practical applications, the $\mathrm{CE}$ with the more diffusive basic scheme is used due to its ability to improve the forecast. Figure 5 also shows that the effective resolution (see, e.g., Skamarock 2004) of the CE applying the basic MPDATA is at least such as that for the CRK model.

The comparison of the CE and CRK vertical velocity spectra (not shown) indicates lower magnitudes of the $\mathrm{CE}$ spectra, especially in the warm season. Further comparison of the vertical velocity and energy spectra in June suggests that the warm season's effect is related to the stronger diffusivity of the CE. However, the CE's improvement of the excessive frequency biases of the CRK forecasts of low and moderate precipitation suggests that the $\mathrm{CE}$ solutions are realistic.

\section{b. Case study for convective weather of 19 July 2013}

The verification experiment indicates that warm-season forecasts particularly profit from the implementation of the EULAG dynamical core. Therefore, several case studies were 

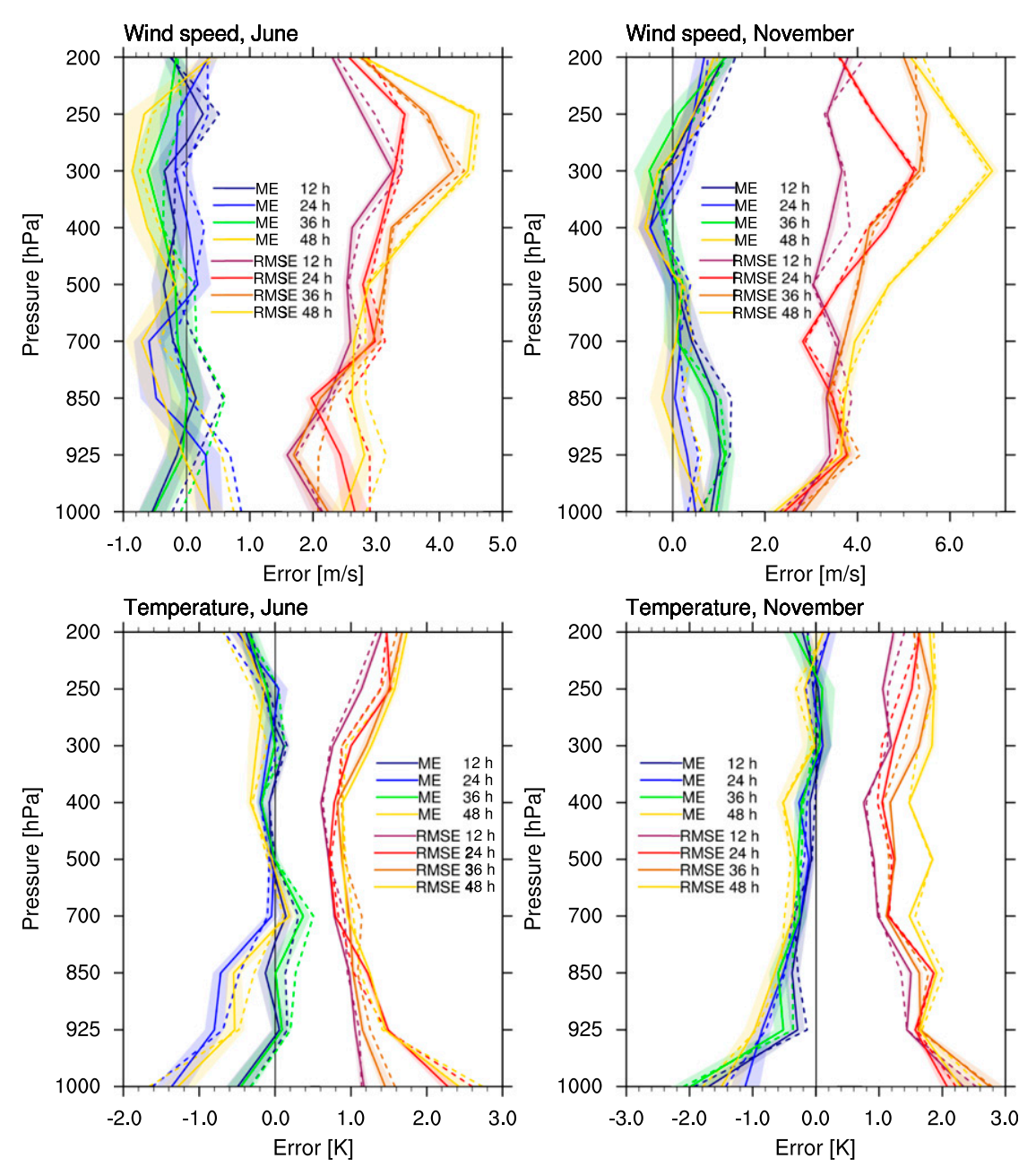

FIG. 7. ME and RMSE for 48-forecast with CE (continuous lines) and CRK (dashed lines) in (left) June and (right) November for (top) upper-air wind speed and (bottom) temperature. The statistical significance of the error differences is indicated as a shaded belt encompassing the characteristics of CE: if the line characterizing the CRK lies outside the belt, the differences are statistically significant with $95 \%$ confidence.

performed for the convectively dominated Alpine weather of 19 July 2013 to test in more detail the CE's ability to represent such weather.

During that day, the Alpine region was covered by a warm air mass outstretched over most of Europe (see Fig. 9 for Met Office surface analysis at 0000 UTC). The flow was weak, and the air mass was convectively unstable with CAPE at upper-air stations varying between $300 \mathrm{~J} \mathrm{~kg}^{-1}$ in Stuttgart, $580 \mathrm{~J} \mathrm{~kg}^{-1}$ in Milan, $950 \mathrm{~J} \mathrm{~kg}^{-1}$ in Payerne, and $1050 \mathrm{~J} \mathrm{~kg}^{-1}$ in Nîmes, at 1200 UTC. The vigorous convective activity took place in the vicinity of the Alps as seen in Fig. 10 presenting Meteosat observations in the HRV (visible) channel.

The experiment employs 24-h CE and CRK forecasts starting at 0000 UTC of that day. The models' configurations and domains are as for the verification tests described above. The focus is on the representations of daytime convective activity reflected by the forecasted cloud fields. The models' clouds, defined by the vertically integrated condensate, including cloud water, ice, snow, and graupel, are compared with HRV Meteosat observations (Fig. 10).

The visual inspection of Fig. 10 indicates that the model clouds and their evolution compare well with the observations. There is also an overall agreement between the models' forecasts. The agreement includes the development of the convection over the Alps, west of them and over the Apennines, and weaker convective activity north of the Alps and over northern Italy. There are also notable differences. In the morning, at 0830 UTC, there are more convective clouds in the CRK forecast, especially in the Alps' vicinity, in better agreement with the observations. Later, when the convection intensifies, the larger spatial density of CE convective clouds over the Alps agrees better with the observations. Also, the lower density of CE convective clouds over Slovenia compares better with the observations.

The cloud forecasts are quantitatively verified using fractions skill scores (FSS; Roberts and Lean 2008). This spatial 

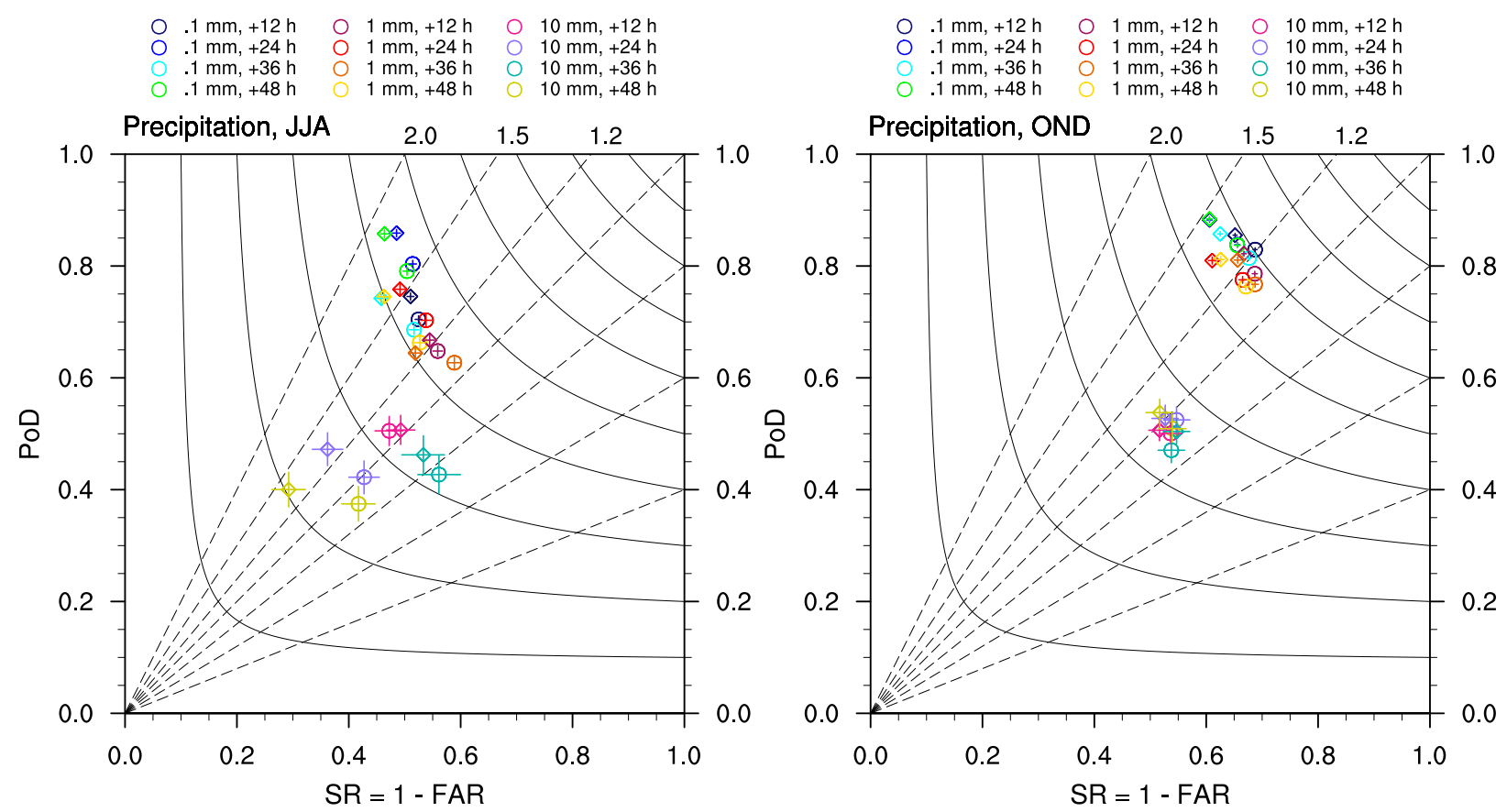

FIG. 8. As in Fig. 4, but for comparison of CE (circles) with the CRK model (diamonds).

method uses a common grid to compare a forecast and observation of a dichotomous event. Here, it is a presence of a cloud at a given grid node, defined by a threshold value of $0.01 \mathrm{~kg} \mathrm{~m}^{-2}$ for vertically integrated cloud condensate of the forecast and of $60 \mathrm{~mW} \mathrm{~m}^{-2} \mathrm{st}^{-1} \mathrm{~cm}^{-1}$ for satellite radiance of the observation. For every node of the grid, the cloud occurrences are counted within its neighborhoods of varying sizes. The neighborhoods have a form of square boxes, with the given node at their center, and their size may vary from the single central grid box to the whole model domain. The forecasting model grid is used for the comparison, and the observations are regridded into it. The skill score is calculated as the mean square error of the forecasted versus observed occurrences, evaluated for a given size of the neighborhood over the whole domain. It is further scaled against a low-skill reference forecast so that the FSS of a perfect forecast is 1 , while 0 indicates a lack of skill.

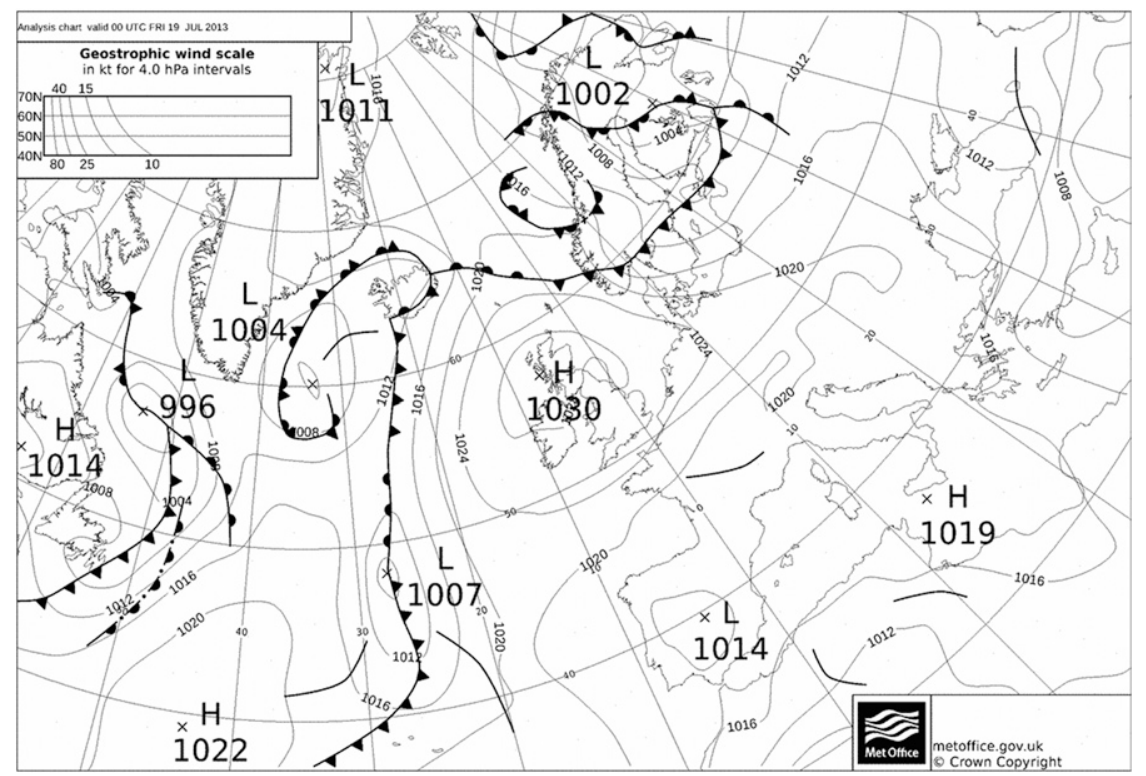

FIG. 9. Met Office surface analysis at 0000 UTC 19 Jul 2013 (U.K. ${ }^{\circ}$ British Crown copyright, Met Office). 


\section{CE clouds}
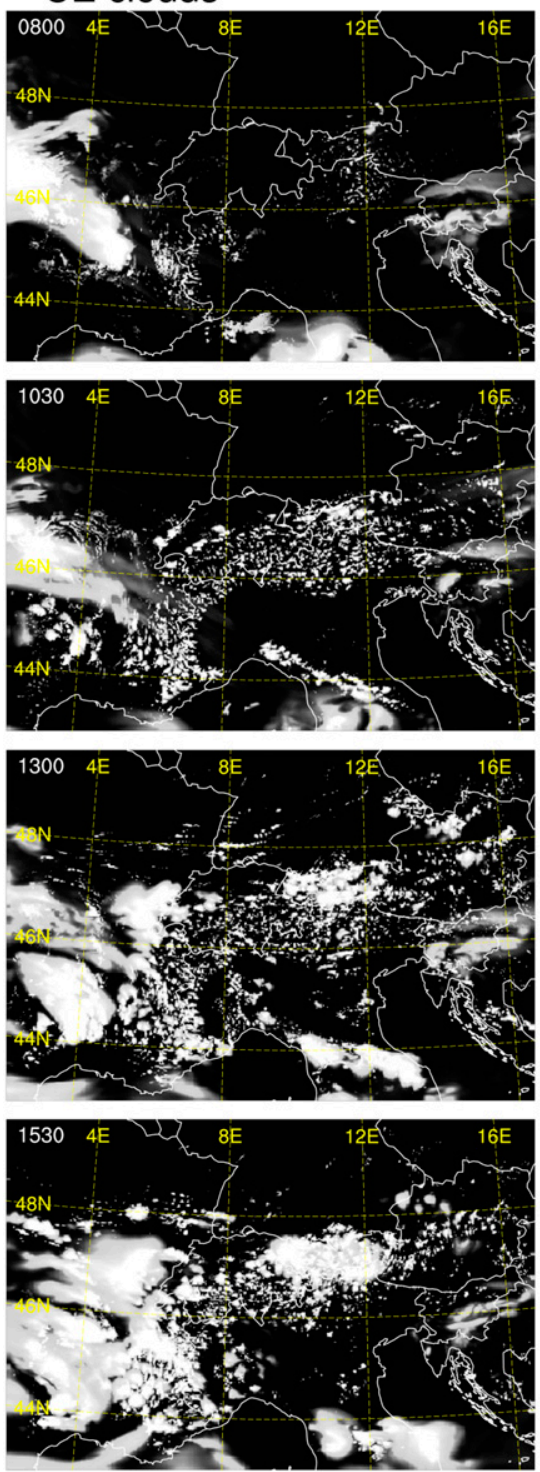

\section{Meteosat HRV}
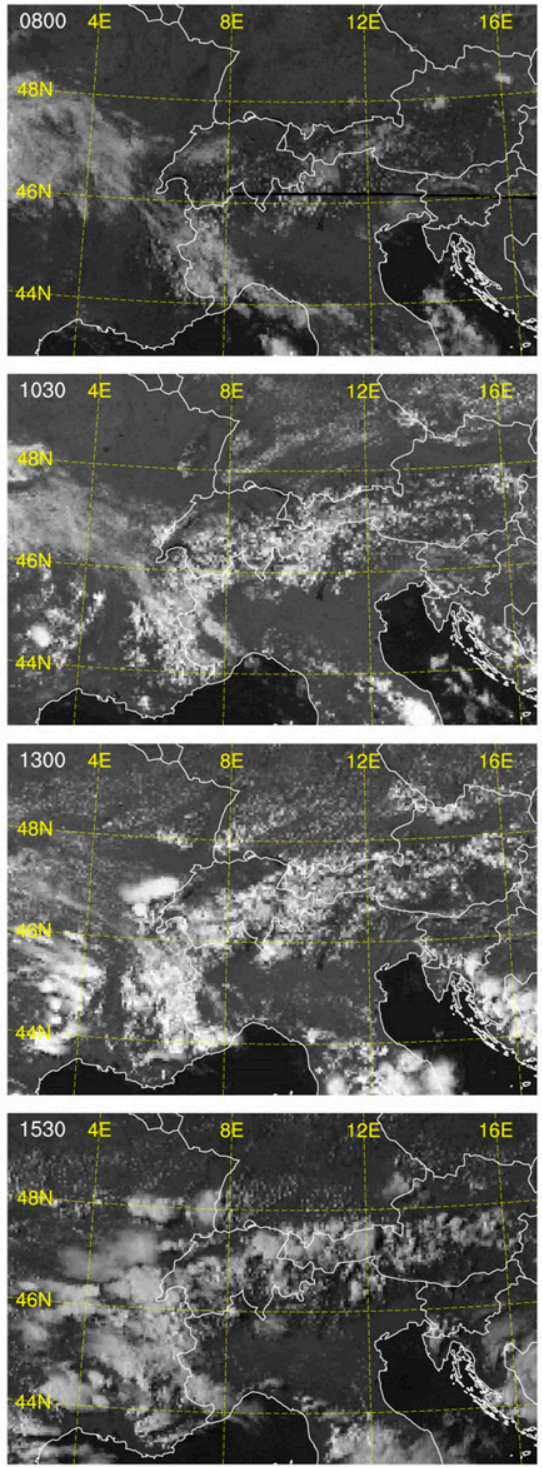

\section{CRK clouds}
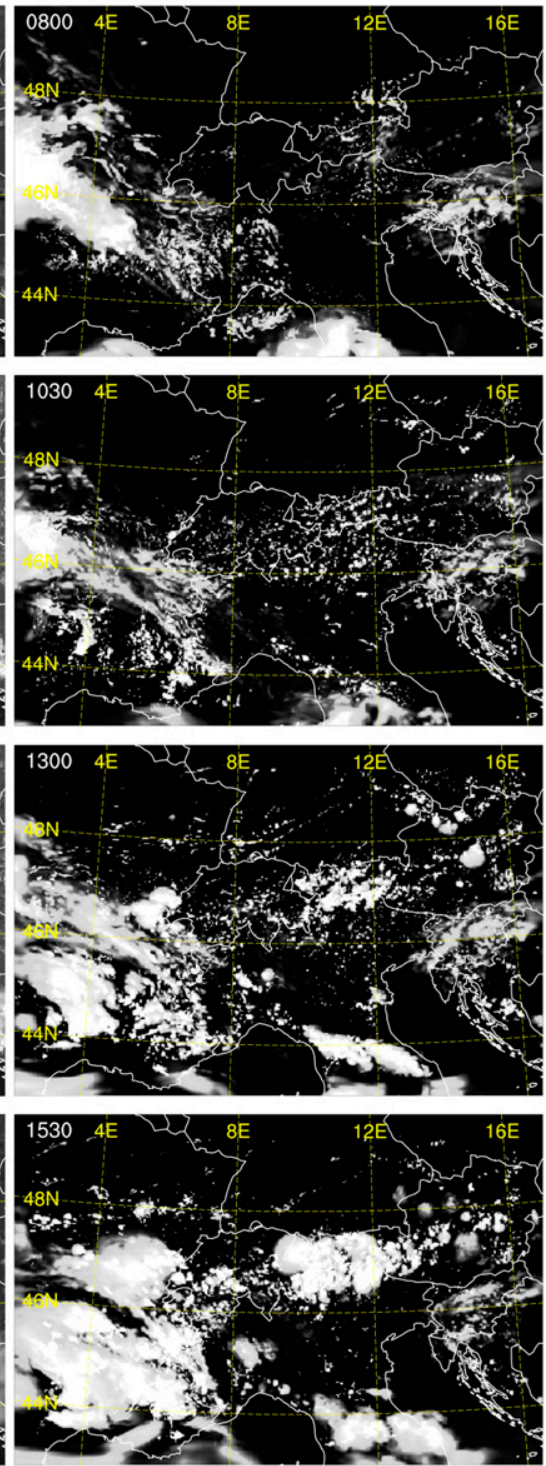

FIG. 10. (center) Meteosat HRV channel over the Alpine domain and its representation by vertically integrated condensate from the (left) CE forecast and the (right) CRK forecast at horizontal grid sizes of $2.2 \mathrm{~km}$ at (top row) 0800 UTC, (second row) $1030 \mathrm{UTC}$, (third row) 1300 UTC, and (bottom row) 1530 UTC.

The satellite observations are provided with a horizontal grid of $5.3 \pm 0.9 \mathrm{~km}$ and are regridded using the nearest neighbor method. The FSS is calculated for neighborhoods of sides' lengths of $1,5,11,21$, and 41 model grid intervals, approximately corresponding to $2,11,24,46$, and $90 \mathrm{~km}$. The threshold for satellite radiances discriminates the cloud field in agreement with a visual inspection of Fig. 10 for most of the day.

Figure 11 compares the evolution of FSS of CE and CRK cloud forecasts, calculated every $15 \mathrm{~min}$ between 0600 and $1800 \mathrm{UTC}$, that is, between 6 and $18 \mathrm{~h}$ of the forecasts. It also shows random FSS (RFSS), uniform FSS (UFSS), and asymptotic FSS (AFSS). Roberts and Lean (2008) define RFSS as FSS calculated at the whole domain scale for a random forecast having the frequency of an event matching the observations' frequency $f_{O}$. The RFSS equals $f_{O}$. The UFSS represents FSS calculated at the gridbox scale if the forecast has the frequency of an event equal to $f_{O}$ at every point. The UFSS equals $0.5+f_{O} / 2$. The FSS increases with the neighborhood box's size, and it finally reaches an AFSS for the neighborhood containing the whole model domain. The AFSS equals $2 f_{B} /\left(1+f_{B}^{2}\right)$, where $f_{B}$ is the frequency bias. Roberts and Lean (2008) argue that the spatial scale at which the FSS becomes larger than the UFSS defines the smallest spatial scale of useful information of the forecast (the smallest useful scale).

Figure 11 indicates very low FSS at the beginning and the end of the analyzed period. It results from a weak illumination of the 
FSS statistics for model clouds

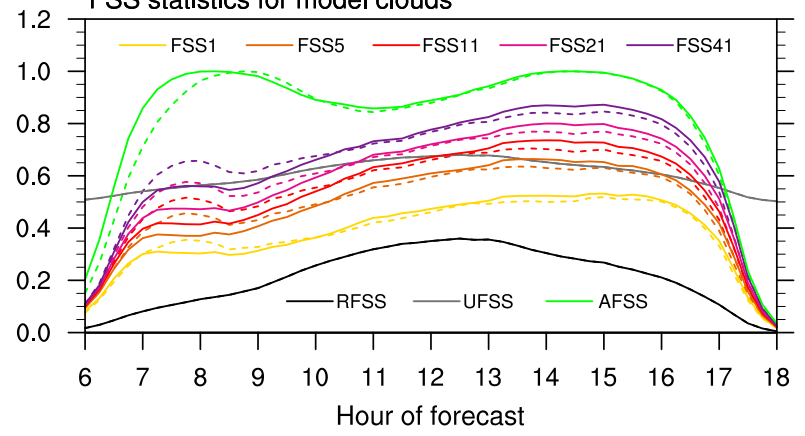

FIG. 11. Evolution of FSS for the CE (continuous line) and CRK (dashed line) cloud fields verified against HRV Meteosat observations; colors indicate the size of the neighborhood boxes at 1,5 , 11,21 , and 41 grid increments. The hour of the forecast is equivalent to the UTC hour of the day.

clouds, leading to their underrepresentation in the dichotomously defined observations. Judging from the AFSS, which after 0700 UTC becomes close to 1 (indicating the frequency bias close to 1 ), the effect ceases at about that time. However, it starts again between 1500 and 1600 UTC when the AFSS decreases significantly. Consequently, further analysis concerns the period between 0800 and 1500 UTC. At the scale of the whole model domain (AFSS), the models' skills are very similar and relatively good. Otherwise, the models have a relatively low skill at 0800 UTC. Later, it systematically increases. In agreement with the visual comparison of observations and forecasts, the CRK skill is at first systematically better at all scales. After about 1000 UTC, the CE skill becomes better at the scales of 2 and $11 \mathrm{~km}$ and similar to the CRK skill at larger scales. After about 1300 UTC, the CE skill becomes better at all scales.

The skill of both models is better than that of a random forecast. However, the smallest useful scale at about 0800 UTC is relatively large and reaches about $40 \mathrm{~km}$ for the CRK and $90 \mathrm{~km}$ for the CE. At about 1030 UTC, both the $\mathrm{CE}$ and CRK smallest useful scales reach $46 \mathrm{~km}$ (after some deterioration, earlier) and systematically improve later. After about 1330 UTC, the scale diminishes below $11 \mathrm{~km}$ for the CE and reaches $11 \mathrm{~km}$ shortly before 1500 UTC for the CRK. In summary, the CE skill measured by FSS and the smallest useful scale is better or neutral to the CRK skill for most of the day, except the morning period.

\section{CE with increased horizontal resolution: A case study}

The experiment extends the previous Alpine case study for the CE forecasts at 1.1-, 0.55-, 0.22-, and 0.1-km horizontal grids, keeping the same vertical grid structure. It practically tests the influence of the increased horizontal resolution on a representation of convective weather, the numerical robustness of the model, and its dynamics-physics coupling. The domain, initial and boundary conditions for simulations at 1.1 - and $0.55-\mathrm{km}$ grids are not changed. Due to computational constraints, the simulations at $0.22-$ and $0.1-\mathrm{km}$ grids have reduced horizontal domains, and their initial and boundary conditions are interpolated from the $\mathrm{CE}$ forecast at a $0.55-\mathrm{km}$ grid.

The same parameterizations of physical processes, except turbulent diffusion, are applied. The simulations using the horizontal kilometer-scale grid, which is comparable with the size of most energetic turbulent eddies, are in the "terra incognita" of turbulence parameterization (Wyngaard 2004). On the one hand, one-dimensional turbulence schemes of mesoscale NWP applications (like the COSMO TKE scheme) assume that the most energetic turbulent eddies are not resolved but contained in the subgrid scales. On the other hand, threedimensional turbulence schemes used for LES-type simulations, usually based on Smagorinsky (1963), assume that the most energetic eddies are resolved, and the schemes are mostly dissipative (see also Shi et al. 2019). In terra incognita, none of these assumptions is valid. Bryan et al. (2003) showed that for deep convection simulations, the horizontal grid increment of $250 \mathrm{~m}$ or smaller is needed to reach the inertial turbulence range and apply LES-type diffusion consistently. In operational practice with the COSMO model at grid intervals of 2.2 and $1.1 \mathrm{~km}$ (e.g., at MeteoSwiss; see Schmidli et al. 2018), the TKE scheme is used with its ability to provide a downgradient transport present in deep convective flows (Verrelle et al. 2017). Following these considerations, the TKE scheme is used for simulations at 2.2 - and $1.1-\mathrm{km}$ grid while at 0.22 - and $0.1-\mathrm{km}$ grid, the three-dimensional Smagorinsky scheme (Langhans et al. 2012; Baldauf and Brdar 2016) is applied. At a $0.55-\mathrm{km}$ grid, both schemes are tested.

The orography and surface parameters for the simulations are generated using the COSMO External Parameters for Numerical Weather Prediction and Climate Application (EXTPAR) software (Asensio et al. 2020). The ASTER (NASA/METI/AIST/Japan Spacesystems and U.S./Japan ASTER Science Team 2019) database for orography, GlobCover (ESA GlobCover 2009 Project 2010) for the land use, Harmonized World Soil Database (HWSD; FAO/IIASA/ISRIC/ISS-CAS/JRC 2012) for soil, and

TABLE 1. CE configuration for forecasts with varying resolution (Smag. is for the Smagorinsky scheme, maximal slope inclination is in degrees).

\begin{tabular}{cccccccc}
\hline \hline $\begin{array}{c}\text { Horizontal grid } \\
\text { size }(\mathrm{km})\end{array}$ & $\begin{array}{c}\text { Zonal } \\
\text { grid No. }\end{array}$ & $\begin{array}{c}\text { Meridional } \\
\text { grid No. }\end{array}$ & $\begin{array}{c}\text { Time } \\
\text { step }(\mathrm{s})\end{array}$ & $\begin{array}{c}\text { BC update } \\
\text { frequency (min) }\end{array}$ & $\begin{array}{c}\text { Simulation } \\
\text { period }\end{array}$ & $\begin{array}{c}\text { Turbulence } \\
\text { scheme }\end{array}$ & $\begin{array}{c}\text { Max. slope } \\
\text { inclination }\end{array}$ \\
\hline 2.2 & 520 & 350 & 12 & 60 & $0000-2400$ UTC & TKE & $21.1^{\circ}$ \\
1.1 & 1040 & 700 & 6 & 60 & $0000-2400$ UTC & TKE & $34.3^{\circ}$ \\
0.55 & 2080 & 1400 & 6 & 60 & $0000-2400$ UTC & TKE, Smag. & $52.5^{\circ}$ \\
0.22 & 1400 & 2080 & 4 & 15 & $0000-1700$ UTC & Smag. & $74.2^{\circ}$ \\
0.1 & 1400 & 2080 & 2 & 15 & $0000-1700$ UTC & Smag. & $85.3^{\circ}$ \\
\hline
\end{tabular}




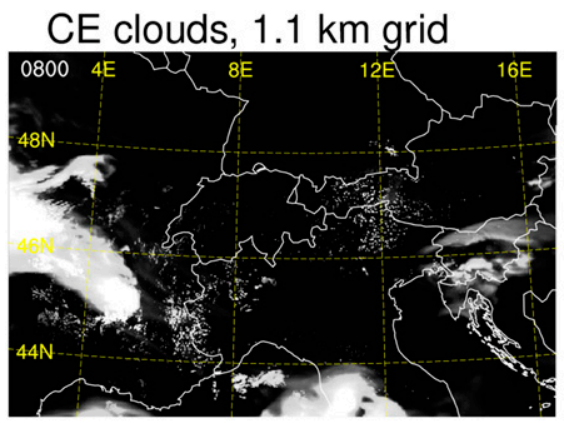

CE clouds, $0.55 \mathrm{~km}$ grid
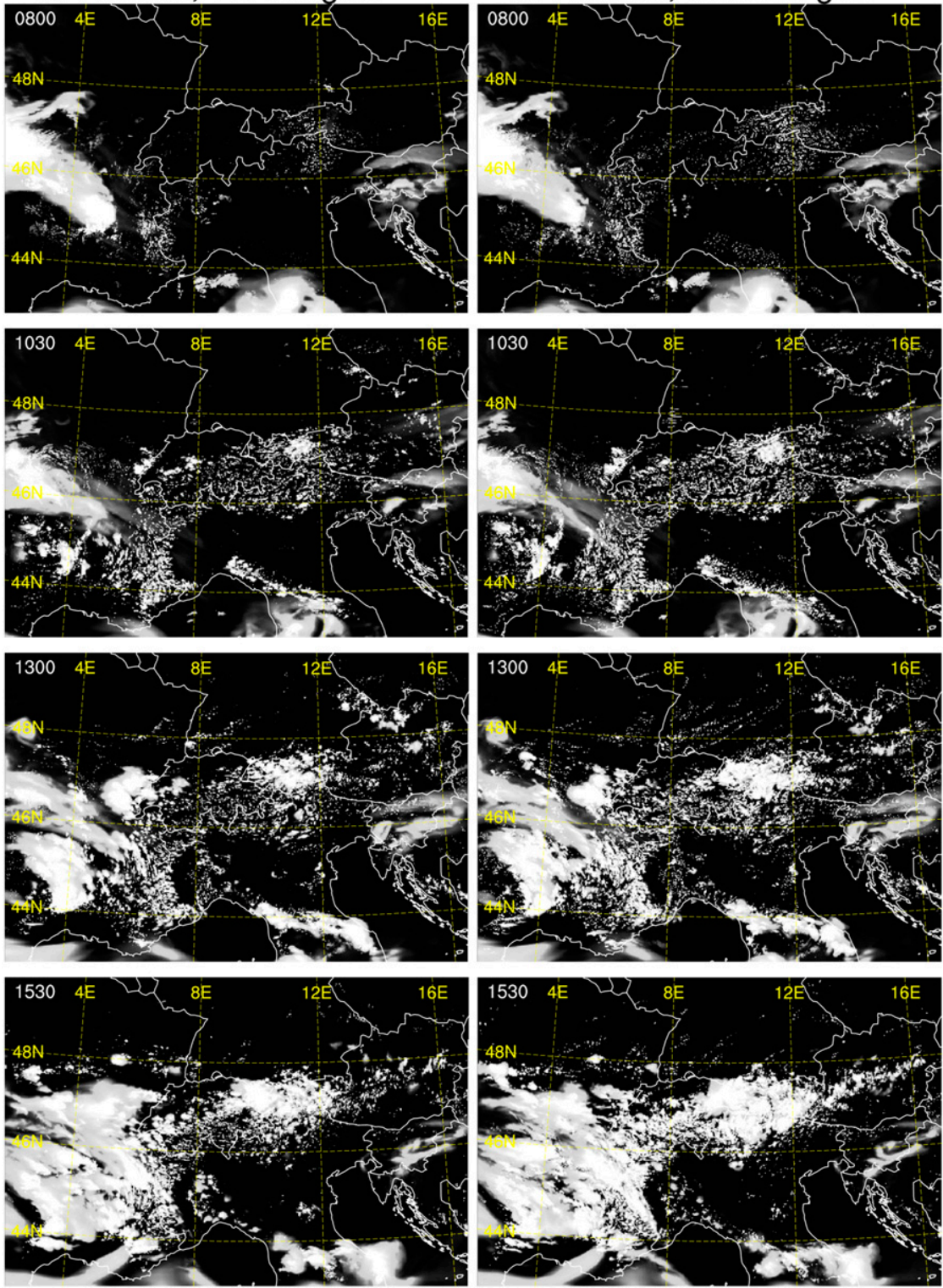

FIG. 12. As in Fig. 10, but for the CE clouds at horizontal grids of (left) $1.1 \mathrm{~km}$ and (right) $0.55 \mathrm{~km}$.

MODIS (Schaaf and Wang 2015) for the albedo are used. The details of model configurations used for varying resolutions, including the maximum inclinations of mountain slopes in the domain, are summarized in Table 1.

\section{a. Convective clouds in CE with 2.2-, 1.1-, and 0.55-km grid}

Figure 12 shows the CE forecasts of convective clouds, represented by vertically integrated condensate, at $1.1-$ and $0.55-\mathrm{km}$ grids. The latter uses the Smagorinsky diffusion. The forecast at 2.2-km grid is in Fig. 10. A comparison of the figures shows a similar spatial distribution and evolution of clouds. The main difference is the morning more abundant presence of the clouds in forecasts at 1.1 and $0.55 \mathrm{~km}$, in agreement with observations
(Fig. 10). At 0800 UTC, it concerns, for example, the western part of Austria, and at 1030 UTC, the whole Alps (see also Fig. 13). The difference is larger between forecasts at 2.2 and $1.1 \mathrm{~km}$ and smaller between forecasts at $1.1-$ and $0.55-\mathrm{km}$ grid. The cloud cover densities at $0.55-\mathrm{km}$ grid are similar for TKE and Smagorinsky diffusions, but the stage of convection tends to be more advanced with the Smagorinsky scheme (see also Fig. 13).

In the afternoon, the differences in the clouds' density are less pronounced. However, deep convective clouds tend to be slightly less developed at increased resolutions when the TKE scheme is used. The use of the Smagorinsky scheme at $0.55 \mathrm{~km}$ averts that effect.

The CE forecasts are further verified against the HRV Meteosat observations using the FSS method defined previously, 

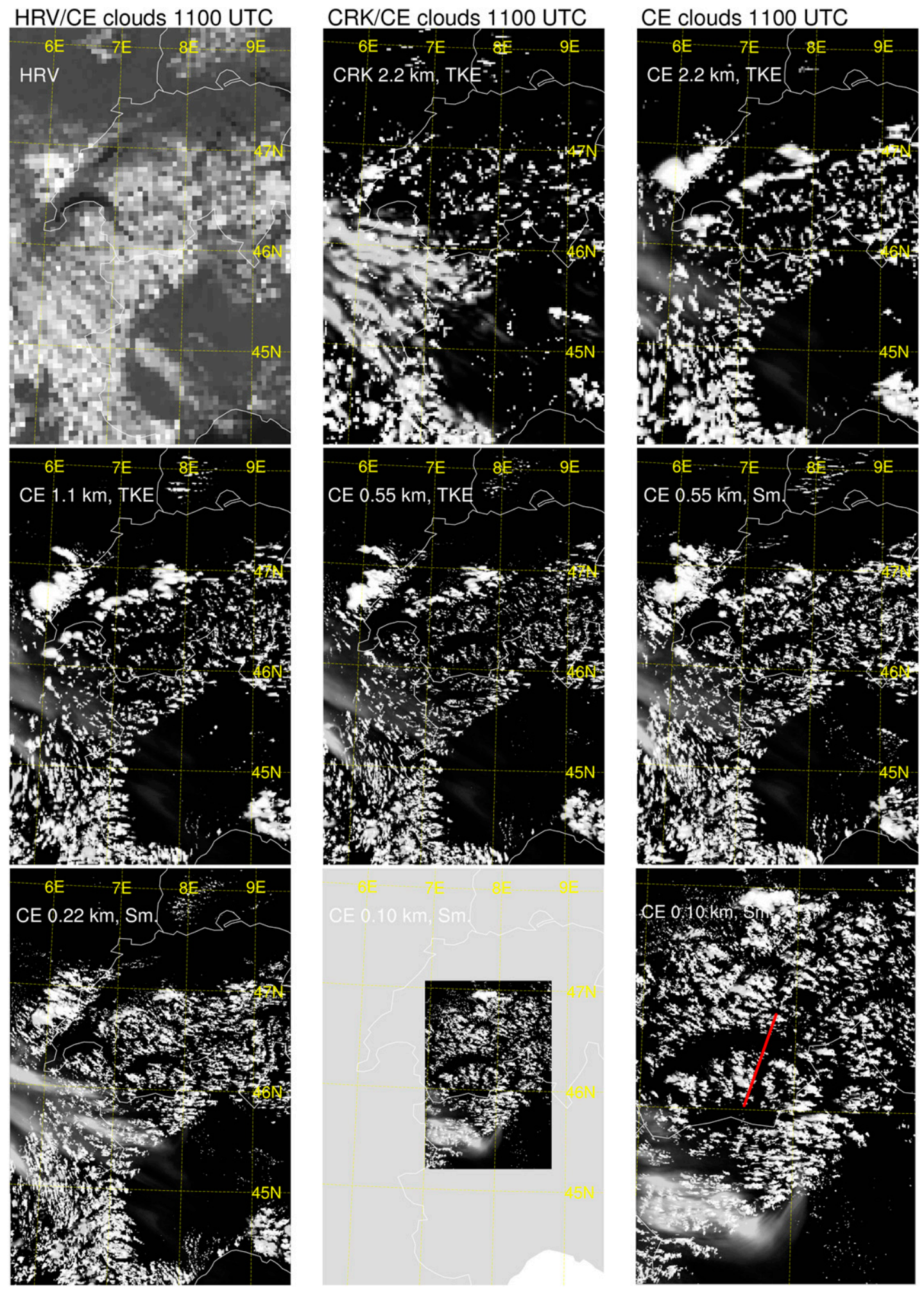

FIG. 13. (top left) Meteosat HRV at 1100 UTC over the CE domain at $0.22-\mathrm{km}$ grid and (top center) its representations by the CRK at 2.2-km grid and the CE at varying grids: (top right) $2.2 \mathrm{~km}$, (middle left) $1.1 \mathrm{~km}$, (middle center) $0.55 \mathrm{~km}$ with TKE diffusion, (middle right) $0.55 \mathrm{~km}$ with Smagorinsky diffusion, (bottom left) $0.22 \mathrm{~km}$, and (bottom center),(bottom right) $0.1 \mathrm{~km}$ (over its domain); red line in the bottom-right panel shows the cross section from Fig. 15. 


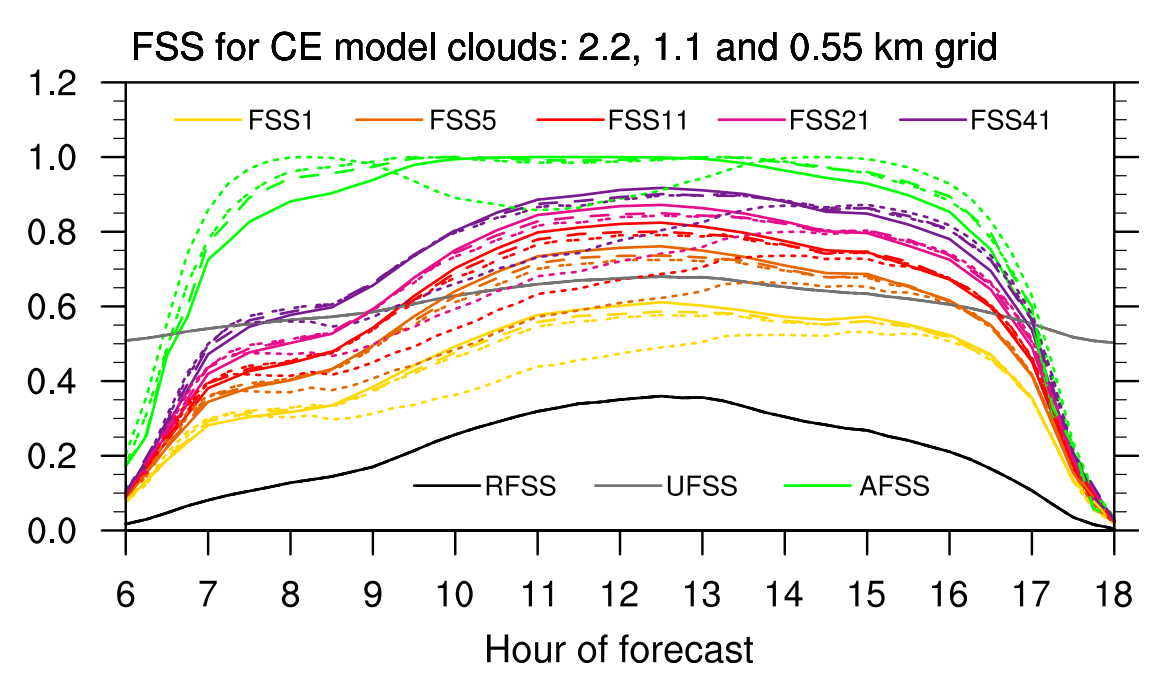

FIG. 14. As in Fig. 11, but for FSS of the CE clouds at the horizontal grid of $2.2 \mathrm{~km}$ (short dash), $1.1 \mathrm{~km}$ (double dash), and $0.55 \mathrm{~km}$ with the TKE diffusion (long dash), and $0.55 \mathrm{~km}$ with the Smagorinsky diffusion (continuous line).

with the clouds at $1.1-$ and $0.55-\mathrm{km}$ grids upscaled to the $2.2-\mathrm{km}$ grid. For the upscaling, two-dimensional horizontal volumes are defined by $2.2-\mathrm{km}$ side boxes centered at every node of the 2.2$\mathrm{km}$ grid. The given volume is considered cloudy if any of the nodes of the 1.1 - or $0.55-\mathrm{km}$ grid located within that volume or on its border is cloudy.

FSS analysis (Fig. 14) indicates that the forecast at 1.1-km grid significantly improves the $2.2-\mathrm{km}$ forecast in the morning and noon hours, at all scales. At $1.1-\mathrm{km}$ forecast, the smallest useful spatial scale improves from about 46 to $11 \mathrm{~km}$ already between 0830 and 1000 UTC. Thus, the smallest useful scale becomes less than $11 \mathrm{~km}$ $3.5 \mathrm{~h}$ earlier than at 2.2-km forecast. Also, the AFSS improves in the late morning and at noon, indicating improved frequency bias of the $1.1-\mathrm{km}$ forecast. The improvement at $0.55 \mathrm{~km}$ compared to the $1.1-\mathrm{km}$ grid is relatively small for the TKE diffusion, but further improvement is provided by the Smagorinsky scheme, especially in the late morning and early afternoon. In the late afternoon, a small deterioration is seen at higher model resolutions at large horizontal scales (above about $24 \mathrm{~km}$ ).

Overall, the CE convective cloud forecasts improve with the increasing horizontal resolution. Further improvement at the $0.55-\mathrm{km}$ grid results from the application of the Smagorinsky scheme. As shown by Verrelle et al. (2015), the three-dimensional Smagorinsky scheme provides a better representation of lateral mixing, which tends to be insufficient within the TKE-based scheme. Sufficiently large lateral mixing is needed to realistically represent the cloud entrainment and detrainment and their influence on cloud development. Also, Tompkins and Semie (2017) demonstrated that sufficient representation of lateral mixing providing adequate entrainment is crucial for appropriate convection-moisture feedback necessary for the realistic organization of deep convection.

b. Convective clouds and vertical velocity at 2.2-0.1-km grid

Additionally, CE forecasts are calculated at 0.22 - and $0.1-\mathrm{km}$ horizontal grids, and the results of all simulations are compared in Fig. 13. It shows the representations of the convective cloud field by the CRK at $2.2-\mathrm{km}$ grid and by the CE at $2.2-0.1-\mathrm{km}$ grid, at 1100 UTC, when the increased horizontal resolution substantially improves the forecast (Fig. 14 for FSS). The clouds are represented by the vertically integrated condensate, shown on the Alpine domain of the CE forecast at the $0.22-\mathrm{km}$ grid, and compared with the HRV observations.

For the CRK and CE forecasts at 2.2-km grid, Fig. 13 confirms a more realistic (dense) distribution of CE clouds mainly over southern Switzerland. The figure shows that with increasing resolution, the CE's clouds spatial density increases further, especially over southern Switzerland, in agreement with the HRV observations. The increase is also seen for a simulation with the $0.22-\mathrm{km}$ grid, while the differences between simulations at 0.22 - and $0.1-\mathrm{km}$ grid are relatively small. The very high-resolution simulations preserve the realistic character of the cloud field.

The further analysis illustrates the influence of increasing resolution on a representation of the mountain flow. Figure 15 shows the vertical cross section through well-developed, mainly thermally driven circulation over the Rhone Valley at 1230 UTC. Vertical velocity simulated by the CRK at $2.2-\mathrm{km}$ grid and by the $\mathrm{CE}$ at the grids varying between 2.2 and $0.1 \mathrm{~km}$ is shown. The cross section cuts between Bietschhorn [3934 $\mathrm{m}$ above the mean sea level (MSL)] on the north (left) and Weisshorn (4506 m MSL) on the south (right) (see Fig. 13, bottom right, for its location).

Figure 15 shows that at $2.2-$ and $1.1-\mathrm{km}$ grid, the flow has a signature of a relatively broad valley circulation with the upward branch along the slopes of the Rhone Valley and the compensating downward branch located immediately above. The horizontal extent of the circulation is wider in the CE and narrower in the CRK simulation. Relatively weak and broad vertical upward currents are associated with the highest peaks and the mountain crest north of Weisshorn. At the $0.55-\mathrm{km}$ grid, the valley circulation fragments, parted by stronger and narrower upward currents associated mainly with local peaks. The currents tend to be stronger when the Smagorinsky diffusion is 

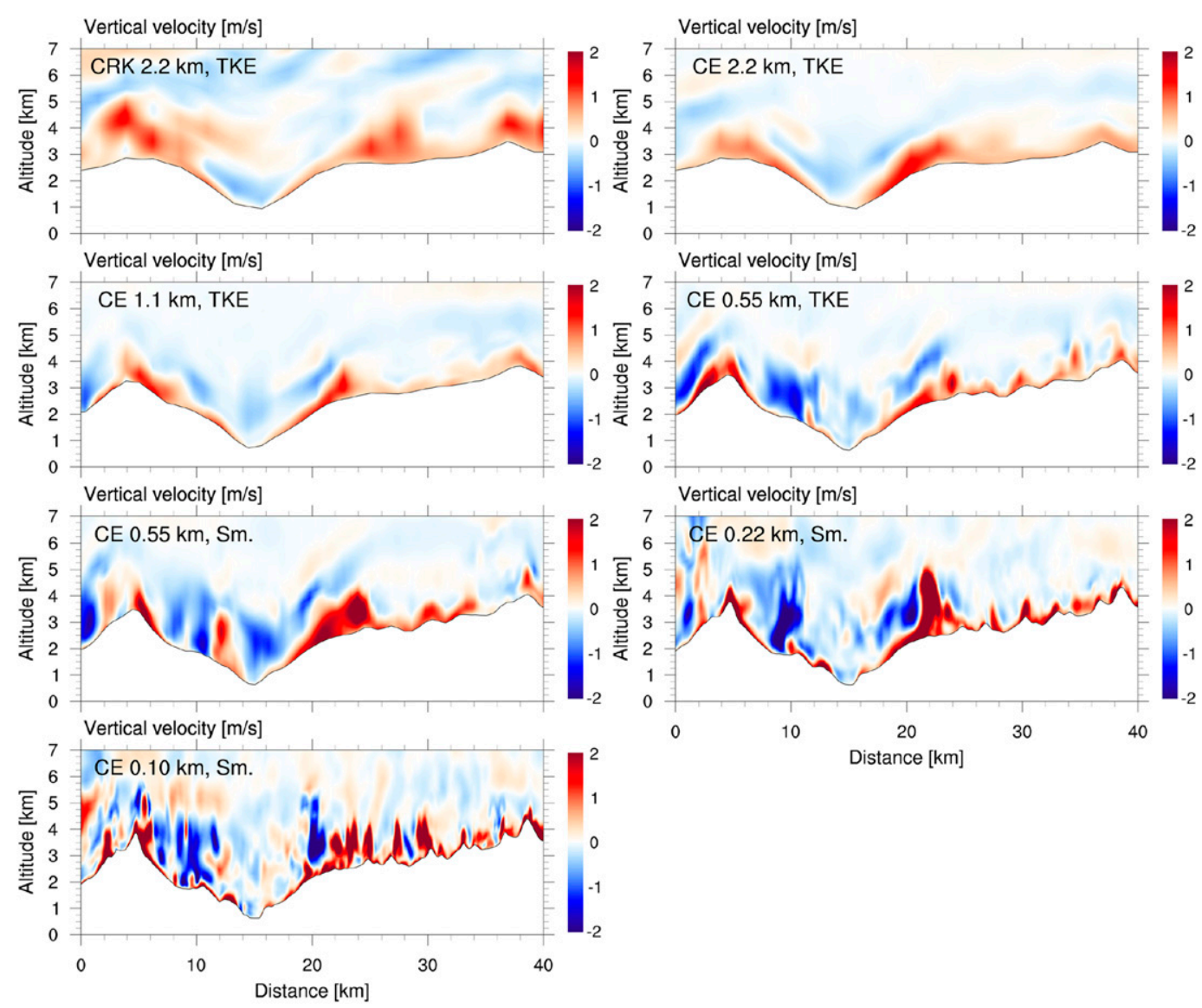

FIG. 15. Vertical velocity $\left(\mathrm{m} \mathrm{s}^{-1}\right)$ at different horizontal grid sizes: vertical cross section from Bietschhorn (the left side of every panel) to Weisshorn (the right side of every panel) at 1230 UTC: (top left) CRK forecast at 2.2-km grid and (top right) CE forecasts at 2.2-km grid, (second row, left) $1.1 \mathrm{~km}$, (second row, right) $0.55 \mathrm{~km}$ with TKE turbulence, (third row, left) $0.55 \mathrm{~km}$ with Smagorinsky turbulence, (third row, right) $0.22 \mathrm{~km}$, and (bottom) $0.1 \mathrm{~km}$.

applied. The further increase of resolution gives more complex orography with local peaks stimulating more numerous and vigorous updrafts, usually associated with compensating downward currents. Such anabatic circulations related to localized heated crests develop in the weak flow regime (see, e.g., a discussion in Demko et al. 2009).

At the cross section, the extremum vertical velocities increase with the resolution up to the $0.22-\mathrm{km}$ grid, but not at the $0.1-\mathrm{km}$ grid. At $2.2-$ and $1.1-\mathrm{km}$ grid, the $\mathrm{CE}$ vertical velocity varies between -1.1 and $1.7 \mathrm{~m} \mathrm{~s}^{-1}$, and for the CRK at 2.2-km grid, it varies between -0.8 and $2.9 \mathrm{~m} \mathrm{~s}^{-1}$. At the 0.55 -km grid, the vertical velocity varies between -1.6 and $3.1 \mathrm{~m} \mathrm{~s}^{-1}$ for the TKE turbulence and between -1.9 and $3.2 \mathrm{~m} \mathrm{~s}^{-1}$ for the Smagorinsky scheme. At the $0.22-\mathrm{km}$ grid, it varies between -3.3 and $7.1 \mathrm{~m} \mathrm{~s}^{-1}$, and at the $0.1-\mathrm{km}$ grid varies between -3.2 and $6.6 \mathrm{~m} \mathrm{~s}^{-1}$. These values, especially at $0.55-\mathrm{km}$ and smaller grids, agree with observations of mountain convection showing localized eddies with vertical velocities of typically $\pm 5 \mathrm{~m} \mathrm{~s}^{-1}$ (Raymond and Wilkening 1980, 1982).

The realistic CE simulations at 0.22 - and $0.1-\mathrm{km}$ horizontal grids with maximum slopes' inclinations exceeding $74^{\circ}$ and almost vertical $85^{\circ}$, respectively (Table 1 ), demonstrate the robustness of the EULAG dynamical core over the complex
Alpine orography to at least 100-m horizontal grid and likely well beyond that threshold. They also indicate a good performance of the model's physics-dynamics coupling.

\section{Conclusions}

The ongoing "quiet revolution" in numerical weather forecasting (Bauer et al. 2015) already allowed for operational regional convective-scale NWP applications. Its further progress sets new, higher demands on the crucial components of the future NWP models, including their dynamical cores. They are expected to provide high accuracy, numerical robustness, conservative properties, and high computational efficiency.

The fully compressible semi-implicit EULAG dynamical core practically implements these requirements, and the paper highlights its first implementation for the realistic convective-scale NWP applications. The paper documents the high accuracy and strong numerical robustness of the dynamical core. Its high computational efficiency was already demonstrated for the IFSFVM implementation (Kühnlein et al. 2019), but the solutions allowing for such efficiency were not yet implemented within the semioperational COSMO-EULAG. 
EULAG employs a numerical approach that does not adhere to the common canons of contemporary computational meteorology and relies on methods historically viewed as problematic (collocated grids, forward-in-time Lax-Wendroff type of advective transport, terrain-following coordinate transformation for very steep slopes, and elaborate elliptic solvers). The work demonstrates that such an approach is competitive to the dominating techniques on staggered grids regarding accuracy and robustness.

The paper considers an optimal choice of EULAG's MPDATA transport scheme for practical NWP applications and shows that its basic version brings overall better verification scores than its gauge version. That is consistent with previous experiences suggesting the basic schemes for simulations of highly turbulent flows. It is hypothesized that the reason lies in potentially higher diffusivity of the basic scheme, better exploring ILES properties of the MPDATA schemes for a physical representation of the SGS processes.

The realistic prognostic simulations of convective Alpine weather with horizontal grid sizes of $2.2,1.1,0.55,0.22$, and $0.1 \mathrm{~km}$ demonstrate the CE's very high-resolution robust representation of weather processes over complex orography, with accuracy growing with its horizontal resolution, at least to $0.55-\mathrm{km}$ grid. The experiment also demonstrates that the increasing resolution allows for a more realistic representation of thermally induced mountain circulations.

Overall, the study shows that the semi-implicit compressible EULAG dynamical core is fit for very high-resolution operational NWP and research applications over complex orography with horizontal grid intervals of at least $100 \mathrm{~m}$. Currently, a project is carried out on the CE's operational application over Poland with a horizontal grid at $\mathcal{O}(1) \mathrm{km}$.

Acknowledgments. The support of Piotr Smolarkiewicz in the implementation of the semi-implicit compressible EULAG is gratefully acknowledged. The work benefitted greatly from an exchange of algorithmic and computational ideas with the developers of the global IFS-FVM solver at ECMWF. The MeteoSwiss kindly provided the initial and boundary conditions for the simulations and the results of meteorological observations for the verification studies. The figures were prepared using NCL software developed at NCAR. Comments of Piotr Smolarkiewicz, Marcin Kurowski, and Andrzej Wyszogrodzki helped improve the manuscript and are gratefully acknowledged. The comments of the editor and three anonymous reviewers helped to improve the paper considerably. Zbigniew Piotrowski acknowledges partial support from the "Numerical weather prediction for sustainable Europe" project, carried out within the FIRST TEAM program of the Foundation for Polish Science co-financed by the European Union under the European Regional Development Fund.

Data availability statement. Codes of COSMO software are the intellectual property of COSMO Members and are not publicly available. The COSMO model code is available under license conditions. Meteosat data are available under EUMETSAT's Data Policy rules. The ASTER, GlobCover, HWSD, and MODIS databases are publicly available. Initial and boundary conditions used for the simulations and observation results used for verification studies are not publicly available due to the regulations of the MeteoSwiss, who provided the data. The model output data for the case-study analyses are available at https://doi.org/10.5281/zenodo.4705290.

\section{REFERENCES}

Arpagaus, M., and Coauthors, 2010: COSMO Science Plan 2010-2014. Consortium for Small-Scale Modeling, 79 pp., https://www.cosmomodel.org/content/consortium/reports/sciencePlan_2010-2014.pdf.

Asensio, H., M. Messmerand, D. Lüthi, and K. Osterried, 2020: External parameters for numerical weather prediction and climate application EXTPAR V.5_0: User and implementation guide. Consortium for Small-Scale Modeling, 45 pp., http://www.cosmomodel.org/content/support/software/ethz/EXTPAR_user_and_ implementation_manual_202003.pdf.

Baldauf, M., and S. Brdar, 2016: 3D diffusion in terrain-following coordinates: Testing and stability of horizontally explicit, vertically implicit discretizations. Quart. J. Roy. Meteor. Soc., 142, 2087-2101, https://doi.org/10.1002/qj.2805.

, A. Seifert, J. Förstner, D. Majewski, M. Raschendorfer, and T. Reinhardt, 2011: Operational convective-scale numerical weather prediction with the COSMO model: Description and sensitivities. Mon. Wea. Rev., 139, 3887-3905, https://doi.org/ 10.1175/MWR-D-10-05013.1.

, and Coauthors, 2013: The COSMO priority project conservative dynamical core final report. Consortium for Small-Scale Modeling, 118 pp., https://doi.org/10.5676/DWD_pub/nwv/cosmo-tr_23.

Bauer, P., A. J. Thorpe, and G. Brunet, 2015: The quiet revolution of numerical weather prediction. Nature, 525, 47-55, https:// doi.org/10.1038/nature14956.

Brown, A. R., M. K. MacVean, and P. J. Mason, 2000: The effects of numerical dissipation in large eddy simulations. J. Atmos. Sci., 57, 3337-3348, https://doi.org/10.1175/1520-0469(2000) 057<3337:TEONDI >2.0.CO;2.

Bryan, G. H., J. C. Wyngaard, and J. M. Fritsch, 2003: Resolution requirements for the simulation of deep moist convection. Mon. Wea. Rev., 131, 2394-2416, https://doi.org/10.1175/15200493(2003)131<2394:RRFTSO>2.0.CO;2.

Clark, T. L., 1977: A small-scale dynamic model using a terrainfollowing coordinate transformation. J. Comput. Phys., 24, 186-216, https://doi.org/10.1016/0021-9991(77)90057-2.

Demko, C. J., B. Geerts, Q. Miao, and J. A. Zehnder, 2009: Boundary layer energy transport and cumulus development over a heated mountain: An observational study. Mon. Wea. Rev., 137, 447-468, https://doi.org/10.1175/ 2008MWR2467.1.

Domaradzki, J. A., Z. Xiao, and P. K. Smolarkiewicz, 2003: Effective eddy viscosities in implicit large eddy simulations of turbulent flows. Phys. Fluids, 15, 3890-3893, https://doi.org/ 10.1063/1.1624610.

Doms, G., and M. Baldauf, 2018: A description of the nonhydrostatic regional COSMO-model: Part I: Dynamics and numerics. Consortium for Small-Scale Modeling, 161 pp., https://doi.org/10.5676/DWD_pub/nwv/cosmo-doc_5.00_I.

_ , and Coauthors, 2018: A description of the nonhydrostatic regional COSMO-model: Part II: Physical parameterization. Consortium for Small-Scale Modeling, 167 pp., https://doi.org/ 10.5676/DWD_pub/nwv/cosmo-doc_5.05_II.

Eisenstat, S. C., H. C. Elman, and M. H. Schultz, 1983: Variational iterative methods for nonsymmetric systems of linear equations. SIAM J. Numer. Anal., 20, 345-357, https://doi.org/ $10.1137 / 0720023$. 
Emanuel, K. A., 1994: Atmospheric Convection. Oxford University Press, $580 \mathrm{pp}$.

ESA GlobCover 2009 Project, 2010: Global Land Cover Map. ESA and UCLouvain, accessed 25 June 2020, http://due.esrin.esa.int/page_ globcover.php.

FAO/IIASA/ISRIC/ISS-CAS/JRC, 2012: Harmonized World Soil Database (version 1.2). FAO and IIASA, accessed 25 June 2020, www.fao.org/soils-portal/soil-survey/soil-maps-anddatabases/harmonized-world-soil-database-v12/en/.

Gal-Chen, T., and R. C. J. Somerville, 1975: On the use of a coordinate transformation for the solution of the Navier-Stokes equations. J. Comput. Phys., 17, 209-228, https://doi.org/ 10.1016/0021-9991(75)90037-6.

Grinstein, F. F., L. G. Margolin, and W. J. Rider, Eds., 2007: Implicit Large Eddy Simulation: Computing Turbulent Fluid Dynamics. Cambridge University Press, $552 \mathrm{pp}$.

Heise, W., M. Lange, B. Ritter, and R. Schrodin, 2003: Improvement and validation of the multi-layer soil model. COSMO Newsletter, No. 3, Consortium for Small-Scale Modeling, Offenbach, Germany, 198-203.

Jaruga, A., S. Arabas, D. Jarecka, H. Pawłowska, P. K. Smolarkiewicz, and M. Waruszewski, 2015: libmpdata ++ 1.0: A library of parallel MPDATA solvers for systems of generalised transport equations. Geosci. Model Dev., 8, 1005-1032, https://doi.org/ 10.5194/gmd-8-1005-2015.

Jensen, J. H., 2017: Which method is more accurate? Or errors have error bars. PeerJ Preprints, 5, e2693v1, https://doi.org/10.7287/ peerj.preprints.2693v1.

Klemp, J. B., and R. Wilhelmson, 1978: The simulation of threedimensional convective storm dynamics. J. Atmos. Sci., 35, 1070-1096, https://doi.org/10.1175/1520-0469(1978)035<1070: TSOTDC $>2.0 . \mathrm{CO} ; 2$.

Kühnlein, C., W. Deconinck, R. Klein, S. Malardel, Z. P. Piotrowski, P. K. Smolarkiewicz, J. Szmelter, and N. P. Wedi, 2019: FVM 1.0: A nonhydrostatic finite-volume dynamical core for IFS. Geosci. Model Dev., 12, 651-676, https://doi.org/10.5194/gmd12-651-2019.

Kurowski, M. J., B. Rosa, and M. Z. Ziemiański, 2011: Testing the anelastic nonhydrostatic model EULAG as a prospective dynamical core of a numerical weather prediction model. Part II: Simulations of supercell. Acta Geophys., 59, 1267-1293, https://doi.org/10.2478/s11600-011-0051-z.

— , P. K. Smolarkiewicz, and W. W. Grabowski, 2014: Anelastic and compressible simulations of moist deep convection. J. Atmos. Sci., 71, 3767-3787, https://doi.org/10.1175/JAS-D14-0017.1.

— W. W. Grabowski, and P. K. Smolarkiewicz, 2015: Anelastic and compressible simulations of moist dynamics at planetary scales. J. Atmos. Sci., 72, 3975-3995, https://doi.org/10.1175/ JAS-D-15-0107.1.

— D. K. Wójcik, M. Z. Ziemiański, B. Rosa, and Z. P. Piotrowski, 2016: Convection-permitting regional weather modeling with COSMO-EULAG: Compressible and anelastic solutions for a typical westerly flow over the Alps. Mon. Wea. Rev., 144, 19611982, https://doi.org/10.1175/MWR-D-15-0264.1.

Langhans, W., J. Schmidli, and B. Szintai, 2012: A Smagorinsky-Lilly turbulence closure for COSMO-LES: Implementation and comparison to ARPS. COSMO Newsletter, No. 12, Consortium for Small-Scale Modeling, Offenbach, Germany, 20-31, http:// www.cosmo-model.org/content/model/documentation/newsLetters/ newsLetter12/2-langhans.pdf.

—_, J. Müller, and W. D. Collins, 2019: Optimization of the eddy-diffusivity/mass-flux shallow cumulus and boundary-layer parameterization using surrogate models. J. Adv. Model. Earth Syst., 11, 402-416, https://doi.org/10.1029/2018MS001449.

LeVeque, R. J., 2002: Finite Volume Methods for Hyperbolic Problems. Cambridge University Press, $580 \mathrm{pp}$.

Lipps, F., and R. Hemler, 1982: A scale analysis of deep moist convection and some related numerical calculations. J. Atmos. Sci., 39, 2192-2210, https://doi.org/10.1175/1520-0469(1982) $039<2192$ :ASAODM $>2.0 . \mathrm{CO} ; 2$.

Lock, S.-J., 2014: Horizontally-explicit vertically-implicit (HEVI) time-stepping methods for NWP and climate models. Seminar on Recent Developments in Numerical Methods for Atmosphere and Ocean Modelling, ECMWF, 201-212, https://www.ecmwf.int/sites/ default/files/elibrary/2014/10771-horizontally-explicit-verticallyimplicit-time-stepping-methods-nwp-and-climate-models.pdf.

Margolin, L. G., P. K. Smolarkiewicz, and Z. Sorbjan, 1999: Largeeddy simulations of convective boundary layers using nonoscillatory differencing. Physica D, 133, 390-397, https:// doi.org/10.1016/S0167-2789(99)00083-4.

Mellor, G. L., and T. Yamada, 1982: Development of a turbulence closure model for geophysical fluid problems. Rev. Geophys. Space Phys., 20, 851-875, https://doi.org/10.1029/RG020i004p00851.

Mengaldo, G., A. Wyszogrodzki, M. Diamantakis, S.-J. Lock, F. X. Giraldo, and N. P. Wedi, 2019: Current and emerging timeintegration strategies in global numerical weather and climate prediction. Arch. Comput. Methods Eng., 26, 663-684, https:// doi.org/10.1007/s11831-018-9261-8.

Moin, P., and A. G. Kravchenko, 1998: Numerical issues in large eddy simulations of turbulent flows. Numerical Methods for Fluid Dynamics VI, M. J. Baines, Ed., ICFD, 123-136.

NASA/METI/AIST/Japan Spacesystems and U.S./Japan ASTER Science Team, 2019: ASTER Global Digital Elevation Model V003. NASA EOSDIS Land Processes DAAC, accessed 25 June 2020, https://doi.org/10.5067/ASTER/ASTGTM.003.

Nicholls, A., 2014: Confidence limits, error bars and method comparison in molecular modeling. Part 1: The calculation of confidence intervals. J. Comput. Aided Mol. Des., 28, 887-918, https://doi.org/10.1007/s10822-014-9753-z.

_ 2016: Confidence limits, error bars and method comparison in molecular modeling. Part 2: Comparing methods. J. Comput. Aided Mol. Des., 30, 103-126, https://doi.org/10.1007/s10822-016-9904-5.

Piotrowski, Z. P., P. K. Smolarkiewicz, S. P. Malinowski, and A. A. Wyszogrodzki, 2009: On numerical realizability of thermal convection. J. Comput. Phys., 228, 6268-6290, https://doi.org/ 10.1016/j.jcp.2009.05.023.

Prusa, J. M., and P. K. Smolarkiewicz, 2003: An all-scale anelastic model for geophysical flows: Dynamic grid deformation. J. Comput. Phys., 190, 601-622, https://doi.org/10.1016/S0021-9991(03)00299-7.

,- , and A. A. Wyszogrodzki, 2008: EULAG, a computational model for multiscale flows. Comput. Fluids, 37, 11931207, https://doi.org/10.1016/j.compfluid.2007.12.001.

Raschendorfer, M., 2001: The new turbulence parameterization of LM. COSMO Newsletter, No. 1, Consortium for Small-Scale Modeling, Offenbach, Germany, 89-98, http://www.cosmomodel.org/content/model/documentation/newsLetters/newsLetter01/ newsLetter_01.pdf.

Raspanti, A., 2009: COSMO Priority Project: "VERification System Unified Survey (VERSUS)": Final report. Consortium for Small-Scale Modeling, 12 pp., https://doi.org/10.5676/DWD_ pub/nwv/cosmo-tr_12.

Raymond, D., and M. Wilkening, 1980: Mountain-induced convection under fair weather conditions. J. Atmos. Sci., 37, 2693-2706, https://doi.org/10.1175/1520-0469(1980) 037<2693:MICUFW >2.0.CO;2. 
_ and - 1982: Flow and mixing in New Mexico mountain cumuli. J. Atmos. Sci., 39, 2211-2228, https://doi.org/10.1175/ 1520-0469(1982)039<2211:FAMINM > 2.0.CO;2.

R Core Team, 2015: R: A Language and Environment for Statistical Computing. R Foundation for Statistical Computing, 201 pp., https://www.R-project.org/.

Reinhardt, T., and A. Seifert, 2006: A three-category ice scheme for LMK. COSMO Newsletter, No. 6, Consortium for Small-Scale Modeling, Offenbach, Germany, 115-120, http://www.cosmo-model.org/content/model/documentation/ newsLetters/newsLetter06/newsLetter_06.pdf.

Ritter, B., and J. F. Geleyn, 1992: A comprehensive radiation scheme for numerical weather prediction models with potential applications in climate simulations. Mon. Wea. Rev., 120, 303-325, https://doi.org/10.1175/1520-0493(1992) $120<0303$ :ACRSFN $>2.0$. CO;2.

Roberts, N. M., and H. W. Lean, 2008: Scale-selective verification of rainfall accumulations from high-resolution forecasts of convective events. Mon. Wea. Rev., 136, 78-97, https://doi.org/ 10.1175/2007MWR2123.1.

Roches, A., and O. Fuhrer, 2012: Tracer module in the COSMO model. Consortium for Small-Scale Modeling, 42 pp., https:// doi.org/10.5676/DWD_pub/nwv/cosmo-tr_20.

Roebber, P. J., 2009: Visualizing multiple measures of forecast quality. Wea. Forecasting, 24, 601-608, https://doi.org/10.1175/ 2008WAF2222159.1.

Rosa, B., M. J. Kurowski, and M. Z. Ziemiański, 2011: Testing the anelastic nonhydrostatic model EULAG as a prospective dynamical core of a numerical weather prediction model. Part I: Dry benchmarks. Acta Geophys., 59, 1236-1266, https://doi.org/ 10.2478/s11600-011-0041-1.

Rossa, A., P. Nurmi, and E. Ebert, 2008: Overview of methods for the verification of quantitative precipitation forecasts. Precipitation: Advances in Measurements, Estimation and Prediction, S. Michaelides, Ed., Springer, 417-450, https:// doi.org/10.1007/978-3-540-77655-0_16.

Schaaf, C., and Z. Wang, 2015: MCD43A1 MODIS/Terra+Aqua BRDF/Albedo Model Parameters Daily L3 Global-500m V006. NASA EOSDIS Land Processes DAAC, accessed 25 June 2020, https://doi.org/10.5067/MODIS/MCD43A1.006.

Schaefer, J. T., 1990: The critical success index as an indicator of warning skill. Wea. Forecasting, 5, 570-575, https://doi.org/ 10.1175/1520-0434(1990)005<0570:TCSIAA > 2.0.CO;2.

Schär, C., D. Leuenberger, O. Fuhrer, D. Lüthi, and C. Girard, 2002: A new terrain-following vertical coordinate formulation for atmospheric prediction models. Mon. Wea. Rev., 130, 2459-2480, https://doi.org/10.1175/1520-0493(2002)130<2459: ANTFVC $>2.0$.CO;2.

Schmidli, J., S. Böing, and O. Fuhrer, 2018: Accuracy of simulated diurnal valley winds in the Swiss Alps: Influence of grid resolution, topography filtering, and land surface datasets. Atmosphere, 9, 196, https://doi.org/10.3390/atmos9050196.

Shi, X., F. K. Chowand, R. L. Street, and G. H. Bryan, 2019: Key elements of turbulence closures for simulating deep convection at kilometer-scale resolution. J. Adv. Model. Earth Syst., 11, 818-838, https://doi.org/10.1029/2018MS001446.

Skamarock, W. C., 2004: Evaluating mesoscale NWP models using kinetic energy spectra. Mon. Wea. Rev., 132, 3019-3032, https://doi.org/10.1175/MWR2830.1.

Smagorinsky, J., 1963: General circulation experiments with the primitive equations: I. The basic experiment. Mon. Wea. Rev., 91, 99-164, https://doi.org/10.1175/1520-0493(1963)091<0099: GCEWTP $>2.3$.CO;2.
Smolarkiewicz, P. K., 1984: A fully multidimensional positive definite advection transport algorithm with small implicit diffusion. J. Comput. Phys., 54, 325-362, https://doi.org/10.1016/0021-9991(84)90121-9.

_- 2006: Multidimensional positive definite advection transport algorithm: An overview. Int. J. Numer. Methods Fluids, 50, 1123-1144, https://doi.org/10.1002/fld.1071.

_ , and T. L. Clark, 1986: The multidimensional positive definite advection transport algorithm: Further development and applications. J. Comput. Phys., 67, 396-438, https://doi.org/ 10.1016/0021-9991(86)90270-6.

— definite advection transport algorithm: Nonoscillatory option. J. Comput. Phys., 86, 355-375, https://doi.org/10.1016/00219991(90)90105-A.

— proximations for fluids. J. Atmos. Sci., 49, 2082-2096, https:// doi.org/10.1175/1520-0469(1992)049<2082:ACOSLA > 2.0.CO;2.

_ for fluids: Extension to curvilinear framework. Mon. Wea. Rev., 121, 1847-1859, https://doi.org/10.1175/1520-0493(1993) 121<1847:OFITDF $>2.0$.CO;2.

- and _ 1994: Variational solver for elliptic problems in atmospheric flows. Appl. Math. Comput. Sci., 4, 527-551.

— geophysical flows. J. Comput. Phys., 140, 459-480, https:// doi.org/10.1006/jcph.1998.5901.

- and - 2000: Variational methods for elliptic problems in fluid models. Proc. ECMWF Workshop on Developments in Numerical Methods for Very High Resolution Global Models, Shinfield Park, Reading, ECMWF, 137-159.

_- and J. M. Prusa, 2002: VLES modelling of geophysical fluids with nonoscillatory forward-in-time schemes. Int. J. Numer. Methods Fluids, 39, 799-819, https://doi.org/10.1002/fld.330.

$\longrightarrow$, and —, 2005: Towards mesh adaptivity for geophysical turbulence: Continuous mapping approach. Int. J. Numer. Methods Fluids, 47, 789-801, https://doi.org/10.1002/fld.858.

_ , and J. Szmelter, 2005: MPDATA: An edge-based unstructured-grid formation. J. Comput. Phys., 206, 624-649, https:// doi.org/10.1016/j.jcp.2004.12.021.

— , V. Grubisić, and L. G. Margolin, 1997: On forward-in-time differencing for fluids: Stopping criteria for iterative solutions of anelastic pressure equations. Mon. Wea. Rev., 125, 647-654, https:// doi.org/10.1175/1520-0493(1997)125<0647:OFITDF>2.0.CO;2.

, L. G. Margolin, and A. A. Wyszogrodzki, 2007a: Implicit largeeddy simulation in meteorology: From boundary layers to climate. J. Fluids Eng., 129, 1533-1539, https://doi.org/10.1115/1.2801678.

—, R. Sharman, J. Weil, S. P. Perry, D. Heist, and G. Bowker, 2007b: Building resolving large-eddy simulations and comparison with wind tunnel experiment. J. Comput. Phys., 227, 633-653, https://doi.org/10.1016/j.jcp.2007.08.005.

_ C. Kühnlein, and N. P. Wedi, 2014: A consistent framework for discrete integrations of soundproof and compressible PDEs of atmospheric dynamics. J. Comput. Phys., 263, 185205, https://doi.org/10.1016/j.jcp.2014.01.031.

— , W. Deconinck, M. Hamrud, C. Kühnlein, G. Mozdzynski, J. Szmelter, and N. P. Wedi, 2016a: A finite-volume module for simulating global all-scale atmospheric flows. J. Comput. Phys., 314, 287-304, https://doi.org/10.1016/j.jcp.2016.03.015.

_ J. Szmelter, and F. Xiao, 2016b: Simulation of all-scale atmospheric dynamics on unstructured meshes. J. Comput. Phys., 322, 267-287, https://doi.org/10.1016/j.jcp.2016.06.048.

_, C. Kühnlein, and N. P. Wedi, 2019: Semi-implicit integrations of perturbation equations for all-scale atmospheric 
dynamics. J. Comput. Phys., 376, 145-159, https://doi.org/ 10.1016/j.jcp.2018.09.032.

Strugarek, A., P. Beaudoin, A. S. Brun, P. Charbonneau, S. Mathis, and P. K. Smolarkiewicz, 2016: Modeling turbulent stellar convection zones: Sub-grid scales effect. Adv. Space Res., 58, 1538-1553, https://doi.org/10.1016/j.asr.2016.05.043.

Tompkins, A. M., and A. G. Semie, 2017: Organization of tropical convection in low vertical wind shears: Role of updraft entrainment. J. Adv. Model. Earth Syst., 9, 1046-1068, https:// doi.org/10.1002/2016MS000802.

Verrelle, A., D. Ricard, and C. Lac, 2015: Sensitivity of highresolution idealized simulations of thunderstorms to horizontal resolution and turbulence parametrization. Quart. J. Roy. Meteor. Soc., 141, 433-448, https://doi.org/10.1002/qj.2363.

,-- , and - 2017: Evaluation and improvement of turbulence parameterization inside deep convective clouds at kilometer-scale resolution. Mon. Wea. Rev., 145, 3947-3967, https://doi.org/10.1175/MWR-D-16-0404.1.

Warn-Varnas, A., J. Hawkins, P. K. Smolarkiewicz, S. A. ChinBing, D. King, and Z. Hallock, 2007: Solitary wave effects north of Strait of Messina. Ocean Modell., 18, 97-121, https:// doi.org/10.1016/j.ocemod.2007.03.003.

Waruszewski, M., C. Kühnlein, H. Pawłowska, and P. K. Smolarkiewicz, 2018: MPDATA: Third order accuracy for variable flows. J. Comput. Phys., 359, 361-379, https:// doi.org/10.1016/j.jcp.2018.01.005.

Wedi, N. P., and P. K. Smolarkiewicz, 2004: Extending Gal-Chen and Somerville terrain-following coordinate transformation on time-dependent curvilinear boundaries. J. Comput. Phys., 193, 1-20, https://doi.org/10.1016/j.jcp.2003.07.034.

Wicker, L. J., and W. C. Skamarock, 2002: Time-splitting methods for elastic models using forward time schemes. Mon. Wea.
Rev., 130, 2088-2097, https://doi.org/10.1175/1520-0493(2002) $130<2088$ :TSMFEM > 2.0.CO;2.

Wójcik, D. K., M. J. Kurowski, B. Rosa, and M. Z. Ziemiański, 2011: A study on parallel performance of EULAG F90/95 code. Parallel Processing and Applied Mathematics: PPAM 2011, R. Wyrzykowski, Eds., Lecture Notes in Computer Science, Vol. 7204, Springer, 419-428, https://doi.org/10.1007/ 978-3-642-31500-8_43.

Wyngaard, J. C., 2004: Toward numerical modeling in the terra incognita. J. Atmos. Sci., 61, 1816-1826, https:// doi.org/10.1175/1520-0469(2004)061<1816:TNMITT $>$ 2.0.CO;2.

Yano, J.-I., and Coauthors, 2018: Scientific challenges of convectivescale numerical weather prediction. Bull. Amer. Meteor. Soc., 99, 699-710, https://doi.org/10.1175/BAMS-D-170125.1.

Zalesak, S. T., 1979: Fully multidimensional flux-corrected transport algorithms for fluids. J. Comput. Phys., 31, 335-362, https://doi.org/10.1016/0021-9991(79)90051-2.

Zängl, G., 2012: Extending the numerical stability limit of terrainfollowing coordinate models over steep slopes. Mon. Wea. Rev., 140, 3722-3733, https://doi.org/10.1175/MWR-D-1200049.1.

Ziemiański, M. Z., M. J. Kurowski, Z. P. Piotrowski, B. Rosa, and O. Fuhrer, 2011: Toward very high horizontal resolution NWP over the Alps: Influence of increasing model resolution on the flow pattern. Acta Geophys., 59, 1205-1235, https://doi.org/ 10.2478/s11600-011-0054-9.

_- and Coauthors, 2015: COSMO Science Plan 2015-2020. Consortium for Small-Scale Modeling, 154 pp., http://www.cosmomodel.org/content/consortium/reports/sciencePlan_20152020.pdf. 